https://helda.helsinki.fi

\title{
Transcription factor PEX1 modulates extracellular matrix turnover through regulation of MMP-9 expression
}

\section{Acosta, Alicia Jurado}

2017-02

Acosta , A J , Rysä , J , Szabo , Z, Moilanen , A-M , Komati , H, Nemer , M \& Ruskoaho , H 2017 , ' Transcription factor PEX1 modulates extracellular matrix turnover through regulation of MMP-9 expression ' , Cell and Tissue Research , vol. 367 , no. 2 , pp. 369-385 . https://doi.org/10.1007/s00441-0

http://hdl.handle.net/10138/309273

https://doi.org/10.1007/s00441-016-2527-2

unspecified

acceptedVersion

Downloaded from Helda, University of Helsinki institutional repository.

This is an electronic reprint of the original article.

This reprint may differ from the original in pagination and typographic detail.

Please cite the original version. 


\title{
Transcription factor PEX1 modulates extracellular matrix turnover through regulation of MMP-9 expression
}

\author{
Alicia Jurado Acosta', Jaana Rysä ${ }^{2}$, Zoltan Szabo', Anne-Mari Moilanen ${ }^{3,4}$, Hiba Komati ${ }^{5}$, \\ Mona Nemer ${ }^{5}$, Heikki Ruskoaho ${ }^{1,6}$
}

${ }^{1}$ Department of Pharmacology and Toxicology, Research Unit of Biomedicine, Faculty of Medicine, University of Oulu, POB 5000, 90014 Oulu, Finland

${ }^{2}$ School of Pharmacy, Faculty of Health Sciences, University of Eastern Finland, POB 1627, 70211 Kuopio, Finland

${ }^{3}$ Cancer and Translational Medicine Research Unit, University of Oulu, POB 5000, 90014 Oulu, Finland

${ }^{4}$ Oulu University Hospital and Medical Research Center Oulu, POB 5000, 90014 Oulu, Finland

${ }^{5}$ Molecular Genetics and Cardiac Regeneration Laboratory, Department of Biochemistry, Microbiology and Immunology, University of Ottawa, 451 Smyth Road, Ottawa, K1H8M5 Ontario, Canada

${ }^{6}$ Division of Pharmacology and Pharmacotherapy, Faculty of Pharmacy, University of Helsinki, POB 56, 00014 Helsinki, Finland

This work was supported by the Finnish Foundation for Cardiovascular Research (to A.J.A., H.R., Z.S.), the Sigrid Jusélius Foundation (to H.R.), the Academy of Finland (grants. 266661 to H.R. and 276747 to J.R.) and the Heart and Stroke Foundation of Canada (grant. NA7301 to H.K. and M.N.). Corresponding author:

Heikki Ruskoaho, MD, PhD

heikki.ruskoaho@helsinki.fi 
Abstract The phenylephrine-induced complex-1 (PEX1) transcription factor, also known as zincfinger protein 260 (Zfp260), is an effector of endothelin-1 and $\alpha_{1}$-adrenergic signaling in cardiac hypertrophy. However, the role of PEX1 in transcriptional regulation of myocardial remodeling remains largely unknown. In the present study, we used PEX1 gain- and loss-of-function to examine the effects of PEX1 on left ventricular remodeling. Adenoviral constructs expressing PEX1, antisense PEX1, or LacZ were delivered by local injection into the anterior wall of the left ventricle in SpragueDawley rats. PEX1 overexpression led to induction of hypertrophic gene program and increased fibrosis. In agreement with this, the expression of genes involved in the fibrotic process, such as collagens I and III, matrix metalloproteinases (MMPs), fibronectin-1, transforming growth factor beta -1 and connective tissue growth factor, were significantly up-regulated following PEX1 overexpression, whereas silencing of PEX1 significantly inhibited the expression of pro-fibrotic genes and increased left ventricular ejection fraction and fractional shortening. In vitro luciferase reporter assays showed that PEX1 regulates the expression of MMP-9 by directly binding to its promoter. Furthermore, PEX1 gain- and loss-of-function experiments in rat neonatal cardiac fibroblasts and myocytes revealed that MMP-9 gene expression was affected by PEX1 predominantly in fibroblasts. Our results indicate that PEX1 is involved in regulating cardiac fibrosis and extracellular matrix turnover, particularly fibroblasts being responsible for the fibrosis-associated changes in gene expression. Furthermore, PEX1 activation of the MMP-9 promoter triggers the profibrotic response directed by PEX1.

Keywords Fibrosis • Hypertrophy $•$ Myocardial remodeling • Signal transduction • Transcription factor 


\section{Introduction}

Left ventricular hypertrophy (LVH) is an adaptive response to pathological conditions, including systemic hypertension, myocardial infarction (MI) and valvular heart disease, which increase workload on the heart (Chan et al. 2012). Although LVH is initiated as a compensatory mechanism to help the myocardium to sustain the mechanical load and ischemic damage, prolongation of this process is maladaptive, leading to significant thickening of ventricular wall and subsequent heart failure, which is the leading cause of morbidity and mortality worldwide (Braunwald 2013).

Extracellular matrix (ECM) alterations are fundamental for the post-MI cardiac remodeling process, which consists of three overlapping stages: inflammation, proliferative or granulation phase, and maturation. At the early stage, there is an acute pro-inflammatory response followed by infiltration of the damaged area by neutrophils and macrophages, as well as activation of fibroblasts (van Nieuwenhoven and Turner 2013; Shinde and Frangogiannis 2014). Cardiac fibroblasts play a major role in ECM maintenance in the adult heart, and increasing evidence indicates that they act as sentinel cells that can be activated under cardiac stress and transform phenotypically into myofibroblasts (MFs) (Fan et al. 2012; van Nieuwenhoven and Turner 2013; Lindner et al. 2014; Shinde and Frangogiannis 2014; Talman and Ruskoaho 2016). Activated fibroblasts migrate to the damaged area and contribute to the healing process. During the proliferative phase, the ischemic tissue is cleared off dead cells and ECM degradation is promoted by increased presence of matrix metalloproteinases (MMPs) to facilitate revascularization (Fan et al. 2012; van Nieuwenhoven and Turner 2013; Shinde and Frangogiannis 2014). At the final stage, anti-inflammatory and pro-fibrotic signaling [such as interleukin-10 (IL-10) and transforming growth factor beta (TGF $\beta$ )] prevails. In response to this, MFs synthesize increasing amounts of ECM structural proteins, such as fibronectin (FN), and type I and III collagens (Serini et al. 1998; Sun et al. 2000; Shinde and Frangogiannis 2014). In addition, MFs express contractile proteins, such as $\alpha$-smooth muscle actin ( $\alpha$-SMA), that allow them to contract the scar and enable wound healing (Sun et al. 2000; Frangogiannis 2000; Shinde and Frangogiannis 2014). Therefore, the primary goal of ECM remodeling is protective, by strengthening the ventricles to sustain a functional myocardium. However, excessive scarring 
increases tissue rigidity and impairs ventricular function, thereby enhancing heart failure progression.

Regardless of the etiology of cardiac remodeling, it involves activation of a complex network of interconnected signaling cascades, which culminate at the nucleus with the activation of a defined set of transcription factors (TF). Many of these TFs have an important role in cardiac development and are re-employed during pathophysiological stress (Oka et al. 2007). In turn, TFs direct the changes in gene expression and re-activation of the fetal gene program, which ultimately leads to structural and functional changes in the heart. The phenylephrine-induced complex-1 (PEX1) TF, also known as zinc-finger protein 260 (Zfp260), is an effector of endothelin-1 and $\alpha_{1}$-adrenergic signaling (Debrus et al. 2005; Komati et al. 2011). Previous studies have shown that PEX1 physically and functionally interacts with GATA4 (Debrus et al. 2005), a master regulator of cardiac development and the hypertrophic response in cardiomyocytes (Pikkarainen et al. 2003; Pikkarainen et al. 2004; Rysä et al. 2010). Specific cardiomyocyte overexpression of PEX1 in vivo and in vitro caused myocyte hypertrophy and induced re-expression of fetal genes associated with the hypertrophic response (Komati et al. 2011). However, the role of PEX1 in transcriptional regulation of cardiac remodeling, especially within non-myocyte cell populations, remains largely unknown.

This study was designed to explore the effects of PEX1 gain- and loss-of-function in the adult heart as a potential modulator of the cardiac ECM remodeling. Here we show that overexpression of PEX1 in the rat left ventricle (LV) induced cardiac fibrosis and up-regulation of fibrosis-associated genes as well as re-activation of the fetal gene program. Gain-of-function experiments in vitro indicated that the pro-fibrotic effects are largely due to transcriptional changes triggered in fibroblasts, and not in cardiomyocytes. Furthermore, promoter binding studies showed that PEX1 directly binds to the MMP-9 promoter, highlighting the role of PEX1 as modulator of ECM turnover during cardiac remodeling. 


\section{Materials and Methods}

\section{PEX1 gene overexpression and silencing}

Recombinant replication-deficient type 5 adenoviruses containing HA-tagged - coding region for PEX1 (Ad5-HA-PEX1) or antisense region directed specifically towards PEX1 (Ad5-AsPEX1) were produced as previously reported (Debrus et al. 2005; Komati et al. 2011). The Ad5-LacZ coding $\beta$ galactosidase LacZ was used as adenoviral control. Predesigned small interference RNA (siRNA) directed against rat PEX1 (NM_017364) and the AllStars Negative Control siRNA were used for in vitro PEX1 silencing experiments. All siRNA constructs and HiPerFect reagent were obtained from Qiagen (Hilden, Germany).

\section{Intramyocardial Gene Transfer}

Male 8-week-old Sprague-Dawley rats (250-300 g) from the colony of the Center of Experimental Animals at the University of Oulu, Finland, were used. Adenovirus-mediated gene transfer was performed as a local intramyocardial injection $\left(1 \times 10^{9} \mathrm{pfu}\right.$ in $100 \mu$ l total volume) with a Hamilton precision syringe directly into the LV free wall as an efficient site-specific method for transgene expression without affecting other organs or other areas of the heart (Rysä et al. 2010). After the operation, anaesthesia was partially antagonized with atipamezole hydrochloride (Antisedan, 1.5 $\mathrm{mg} / \mathrm{kg}$, intraperitoneal) and rats were hydrated with physiological saline solution $(5 \mathrm{ml}$ subcutaneous). For postoperative analgesia, buprenorphine hydrochloride (Vetergesic, 0.05-0.2 $\mathrm{mg} / \mathrm{kg}$, subcutaneous) was administered. All animal procedures were approved by the Animal Use and Care Committee of the University of Oulu and the Provincial Government of Southern Finland Department of Social Affairs and Health and conform to the guidelines from Directive 2010/63/EU of the European Parliament on the protection of animals used for scientific purposes.

\section{Acute Myocardial Infarction}

MI was produced by ligation of the left anterior descending (LAD) coronary artery in anaesthetized rats (Tenhunen et al. 2006b; Rysä et al. 2010). Rats were anesthetized with ketamine (50 mg/kg, 
intrapreritoneal) and xylazine//medetomidine (10 mg/kg, intraperitoneal). The adenoviral gene transfer was performed before LAD ligation.

\section{Angiotensin II-Induced Hypertension}

In a separate series of experiments, angiotensin II (Ang II, 33.3 $\mu \mathrm{g} / \mathrm{kg}$ per hour) was administered via subcutaneously implanted osmotic minipumps for 1 week and 2 weeks (Alzet model 2002, DURECT Corporation, Cupertino, CA, USA) (Serpi et al. 2011). The minipumps were implanted in SD rats under inhaled anesthesia with isoflurane (Baxter International Inc.). Recombinant adenovirus was injected after minipump implantation. In this experimental model of hypertension, the mean arterial pressure increases within 3 hours and it is sustained throughout 2 weeks (Suo et al. 2002).

\section{Echocardiography}

Transthoracic echocardiography was performed using the Vevo2100 high frequency high resolution linear array ultrasound system (FujiFilm VisualSonics Inc, Toronto, Canada) and MS-250 transducer $(13-24 \mathrm{MHz}$, axial resolution $75 \mu \mathrm{m}$, lateral resolution $165 \mu \mathrm{m})$ by a trained sonographer blinded to the treatments. Rats were anesthetized with ketamine $(50 \mathrm{mg} / \mathrm{kg}$, intrapreritoneal) and xylazine (10 $\mathrm{mg} / \mathrm{kg}$, intraperitoneal). Using two-dimensional imaging, a short axis view of the left ventricle at the level of the papillary muscles was obtained, and a two dimensionally guided M-mode recording through the anterior and posterior walls of the LV were acquired. LV end-systolic and end-diastolic dimensions as well as the thickness of the interventricular septum and posterior wall were measured from the M-mode tracings. LV fractional shortening (FS) and ejection fraction (EF) were calculated from the M-mode LV dimensions using the equations 1 and 2 :

$$
\begin{gathered}
\mathrm{FS}(\%)=\left\{(\text { LVEDD }- \text { LVESD }) / \text { LVEDD } \times 100^{(1)}\right. \\
\mathrm{EF}(\%)=\left\{\left(\text { LVEDD }^{3}-\left(\text { LVESD }^{3} / \text { LVEDD }^{3}\right\} \times 100^{(2)}\right.\right.
\end{gathered}
$$

An average of three measurements of each variable was used. After echocardiographical measurements at 3 days, 1 week or 2 weeks, the animals were sacrificed, hearts were excised and tissue samples were processed for histological tests and expression characterization. 


\section{Histology and immunochemistry}

The upper transversal mid-section of the heart was fixed in $10 \%$ buffered formalin solution and then embedded in paraffin. Five $\mu \mathrm{m}$ thick sections were sliced from the mid-section of the heart at the level of the papillary muscles. In order to make the samples fully comparable, samples from different animals were obtained in an identical way and from the corresponding sites. The sections were deparaffinized in xylene and dehydrated in graded ethanol series. Sections were stained with Masson's trichrome and Picrosirius red (Direct Red 80, Sigma-Aldrich, St. Louis, MO, USA). The total fibrotic area of the LV was measured from 5 hotspots in the Masson's trichrome stained sections. Picrosirius red-stained sections were visualized with linear polarized light (Olympus BX51).

Wheat germ agglutin/5'-fluorescein isothiocyanate (WGA-FITC) was used to quantify cardiomyocyte cross sectional area. The cell area was calculated by measuring a total of $10-15$ cells (from 5 LV fields) per heart using Adobe Image CS5 software. Apoptotic cells were determined by terminal deoxynucleotidyl transferase-mediated dUTP nick end labeling (TUNEL) method, according to the manufacturer's protocol (ApopTag Red in situ apoptosis detection kit, Chemicon). Primary antibodies for Ki-67, von Willebrand factor (vWF), and c-kit were used to label proliferating cells, endothelial cells, and cardiac stem cells, respectively.

The efficiency of PEX1 adenoviral gene transfer was confirmed by specific immunodetection of PEX1 with polyclonal anti-PEX1 antibody (Debrus et al. 2005). Immunofluorescent co-staining was used to visualize the cell-specificity of adenoviral PEX1 expression. Specific cell markers for fibroblasts (prolyl-4-hydroxylase, $\mathrm{P} 4 \mathrm{H}$ ) and myocytes ( $\alpha$-actinin) were used in combination with antiPEX1 antibody. Images were generated using 3DHISTECH Pannoramic 250 FLASH II digital slide scanner at the Genome Biology Unit (Research Programs Unit, Faculty of Medicine, University of Helsinki, Biocenter Finland) and the Institute of Biotechnology (University of Helsinki). Table 1 in the Electronic Supplementary Materials contains the details of all the antibodies utilized. 


\section{Primary neonatal cell culture}

The cell culture medium components [Dulbecco's modified Eagle's medium (DMEM)/F-12, fetal bovine serum (FBS) and $100 \mathrm{IU} / \mathrm{ml}$ penicillin-streptomycin, bovine serum albumin, $1 \mu \mathrm{M}$ insulin, $2.8 \mathrm{mM}$ sodium pyruvate, $5.64 \mu \mathrm{g} / \mathrm{ml}$ transferrin, $1 \mathrm{nM} \mathrm{T3}$ ] were all purchased from Sigma-Aldrich (St. Louis, MO). Neonatal rat ventricular myocytes (NRVM) and fibroblasts (NRVF) were prepared from 2- to 4-days-old Sprague-Dawley rats using the collagenase dissociation method as described before (Pikkarainen et al. 2003). The animals were killed by decapitation and the ventricles were excised. Differential attachment preplating was used to separate non-myocytes from myocytes. NRVM were recovered from the unattached fraction and were seeded $\left(1.5-2 \times 10^{5} \mathrm{cells} / \mathrm{cm}^{2}\right)$ in 12 well plates. The attached fraction is enriched with NRVF, which were passaged twice before seeding for the experiment $\left(0.75-1 \times 10^{5}\right.$ cells $/ \mathrm{cm}^{2}$ into 12 -well plates). The cells were maintained in DMEM/F12 supplemented with $10 \%$ FBS and $100 \mathrm{IU} / \mathrm{ml}$ penicillin-streptomycin. After 24 hours, cells were changed to culture serum-free media (CSFM), containing DMEM/F-12 supplemented with $2.5 \mathrm{mg} / \mathrm{ml}$ bovine serum albumin, $1 \mu \mathrm{M}$ insulin, $2.8 \mathrm{mM}$ sodium pyruvate, $5.64 \mu \mathrm{g} / \mathrm{ml}$ transferrin, $1 \mathrm{nM}$ T3, and $100 \mathrm{IU} / \mathrm{ml}$ penicillin-streptomycin.

NRVM or NRVF were infected 18-24 hours after the initial plating with Ad5-HA-PEX1 or Ad5LacZ (MOI 2) for overexpression experiments, and PEX1-siRNA or AllStars Negative Control siRNA were used for silencing experiments. The transfection of synthetic siRNAs was achieved by using HiPerFect reagent according to the manufacturer instructions. Briefly, on the day of transfection, the cells were washed and changed to CSFM. The siRNAs and HiPerFect were diluted in DMEM and gently mixed to achieve the final concentration of $5 \mathrm{nM}$ siRNA and of $4 \mu \mathrm{H} \mathrm{HiPerFect/well,} \mathrm{and}$ incubated at room temperature to allow the formation of siRNA-HiPerFect complexes. Transfection silencing complexes or adenoviruses were added to the cells and incubated at $37^{\circ} \mathrm{C}$ and $5 \% \mathrm{CO}_{2}$. The cell culture medium was replaced every 24 hours. The cells were washed twice with ice-cold PBS and quickly frozen at $-70^{\circ} \mathrm{C}$ after 24 to 72 hours, accordingly to the experimental requirements. 


\section{Isolation and analysis of RNA}

Total RNA from whole tissue of LV lower transversal mid-section was isolated by the guanidine thiocyanate-CsCl method (Rysä et al. 2010). Extraction of RNA from NRVM and NRVF was performed using the E.Z.N.A.® Total RNA Kit I (Omega Bio-Tek Inc. Norcross, GA, USA) according to the manufacturer's instructions. The RNA derived from LV tissue or neonatal cells was used as template to synthesize cDNA using Transcriptor First-Strand cDNA Synthesis Kit (Roche Diagnostics, Mannheim, Germany) according to the manufacturer's instructions. Real-time quantitative polymerase chain reaction (RT-qPCR) with TaqMan chemistry (Life Technologies) was used for RNA expression level analysis on an ABI 7300 sequence detection system (Applied Biosystems, Life Technologies) as previously described (Tenhunen et al. 2006b). The primers and fluorogenic probes utilized for RT-qPCR were all purchased for Sigma Aldrich and are listed in the Electronic Supplementary Material, Table 2.

\section{Protein extraction and western blotting}

Total and nuclear protein extraction for Western blot analyses were performed as previously described (Tenhunen et al. 2006a). Protein levels were detected using fluorescence with Odyssey Fc imaging system (LI-COR Biosciences, Lincoln, NE, USA). The bands were quantified with Image Studio software (LI-COR). Table 1 in the Electronic Supplementary Material contains the details of all the antibodies utilized.

\section{Luciferase reporter assay}

COS-1 cells were maintained in DMEM containing 10\% FBS. The cells were transfected with pGLIII Basic vector coding for beetle luciferase containing 1.8-kb fragment of MMP-9 promoter (pGL-III - pMMP-9 - Luc) (Eberhardt et al. 2002) and different concentrations of pCGN-PEX1 or pCGN empty control plasmid. Transfections were carried out using FUGENE-6 reagent (Promega Corporation, Madison, WI, USA) according to the manufacturer's instructions. The transfections were performed as triplicates and repeated at least three times to ensure reproducibility of the results. Transfection with $\mathrm{pCMV}$ coding for $\beta$-galactosidase was used to control for transfection efficiencies. Luciferase 
and $\beta$-galactosidase activities were measured with Luciferase Assay System kit (Promega Corporation) and Luminescent Beta-galactosidase Detection Kit II (Clontech Laboratories, Inc., Mountain View, CA, USA) using an automated chemiluminescence detector (Varioskan Flash, Thermo Fisher Scientific Oy, Vantaa, Finland)

\section{Data analysis and statistics}

For statistical analysis, data was first tested for normality by using the Shapiro-Wilk test. For normally distributed variables, pairwise comparisons between two groups were made by unpaired two-tailed Student's t-test. For data that was not normally distributed, statistical analysis was performed by using non-parametric Mann-Whitney U-test. Probability values below 0.05 were considered significant. Results are reported as mean \pm standard deviation (SD). Statistical analyses were performed with SPSS software (SPSS Inc., Chicago, IL, USA).

\section{Results}

\section{Augmentation and silencing of PEX1 expression by intramyocardial adenoviral gene transfer} in vivo

To investigate the potential role of PEX1 in cardiac remodeling, rats were subjected to in vivo intramyocardial gene transfer (Tenhunen et al. 2006a; Tenhunen et al. 2006b; Rysä et al. 2010) to locally modulate the LV levels of PEX1. Different doses of Ad5-HA-PEX1 and Ad5-AsPEX1 adenoviral constructs were first tested to ensure up-regulation and down-regulation of LV PEX1 expression, respectively. As shown in Fig. 1a, PEX1 mRNA levels markedly increased at day 3 after the gene transfer in the Ad5-HA-PEX1-injected group when compared to LacZ-injected animals (21.4-fold, $P<0.005)$, remained elevated at 1 week ( 3.6 -fold, $P<0.01$ ), and returned to basal levels 2 weeks post-intervention. On the other hand, silencing of PEX1 expression by adenoviral delivery of AsPEX1 construct resulted in significant down-regulation of PEX1 mRNA levels at 2 weeks after gene transfer (Fig. 1a). The presence of PEX1 in LV was confirmed by immunostaining the heart sections using anti-PEX1 antibody (Fig. 1b-d"). The strongest signal was observed at day 3 in Ad5- 
HA-PEX1-treated hearts (Fig. 1c), mostly localized around the areas of injection, and being almost absent from LacZ- (Fig. 1b-b") and AsPEX1-injected animals (Fig. 1d-d"). To characterize further the efficiency and localization of the PEX1 gene delivery, consecutive histological slices from PEX1 overexpressing hearts at 3 days were used for double immunofluorescent detections of PEX1 with the cell-type specific markers sarcomeric a-actinin for myocytes and P4H for fibroblasts. PEX1 colocalized with a-actinin and $\mathrm{P} 4 \mathrm{H}$, demonstrating that PEX1 overexpression occurred in both cell types, approximately $60 \%$ of PEX1-positive were myocytes and $25 \%$ were fibroblasts (Fig. 2).

\section{PEX1 induces fibrotic process in the adult heart}

Intramyocardial delivery of PEX1 adenoviral construct in the normal rat heart triggered significant augmentation of interstitial fibrosis at 2 weeks, as measured by Masson's trichrome (Fig. 3a, a', b, b', c, c', d) and Picrosirius red stainings (Fig. 3a",b",c"). We also assessed changes in the expression of genes involved in the fibrotic and remodeling processes by RTqPCR from LV tissue extracts. Collagens la1 and IIla1 are major components of the ECM, whose expression was significantly increased $(P<0.01)$ at day 3 in response to PEX1 overexpression (Fig. 3e, f). Interleukin-6 (IL-6), an early mediator of the inflammatory response to stress injury, was also significantly up-regulated at 3 days (Fig. 3g). Interestingly, MMP-9 mRNA levels rose 8.9 -fold at 3 days $(P<0.005)$ and progressively returned to baseline levels at 1 and 2 weeks in response to PEX1 overexpresssion (Fig. 3h). A more moderated and sustained effect on MMP-2 was observed; MMP-2 mRNA levels were 2-fold higher in PEX1 overexpressing hearts than the control group at 3 days and 1 week (Fig. $3 i ; P<0.01$ and $P<0.05$, respectively). Matrix deposition of $F N-1$ is induced by TGF $\beta 1$ and promoted by connective tissue growth factor (CTGF) (Leask and Abraham 2004), which is also a mediator of TGF 1 actions (Chen et al. 2000; Abreu et al. 2002; Garrett et al. 2004; Leask and Abraham 2004). While FN-1 mRNA levels remained substantially elevated at 3 days and 1 week after gene transfer (Fig. 3j), the expression of TGF $\beta 1$ and CTGF were increased at 3 days and returned to basal levels after 1 week (Fig. 3k, I). Similarly, a-SMA (also known as Acta2), a well-established marker for cardiac fibroblast to myofibroblasts transdifferentiation, followed the same expression pattern as pro-

fibrotic genes being significantly up-regulated by PEX1 overexpression at 3 days (Fig. 3m). 
Furthermore, a-SMA was localized with PEX1 (Electronic Supplementary Material, Fig. S1). On the other hand, silencing of PEX1 expression by AsPEX1 adenoviral construct at 2 weeks had an opposite effect on the transcriptional regulation by causing significant reduction of mRNA levels of genes typically activated during the ECM remodeling and fibrotic process (Fig. 3e-m).

\section{Cell-specific actions of PEX1 on fibrosis-related gene expression}

Since PEX1 co-localized with $\alpha$-actinin and P4H in the adult heart, we next studied whether PEX1 has differential effects on gene expression in fibroblasts and myocytes. Gain- and loss-of-function studies were conducted in vitro using NRVM and NRVF harvested from 2-4 days old rats and were transfected with Ad5-HA-PEX1 or PEX1-siRNA for overexpression or silencing studies, respectively. As shown in Fig. 4, PEX1 overexpression at 24 and 48 hours markedly increased MMP-2 and MMP9 mRNA levels in NRVFs, while no significant changes were observed in NRVMs. Similarly, the proinflammatory cytokine IL-6 mRNA levels were significantly increased in NRVF. Interestingly, a-SMA and CTGF mRNA levels were down-regulated in NRVF, but not in NRVM, whereas changes in mRNA levels of FN-1 (Fig. 4), collagens and other profibrotic markers (data not shown) explored were not statistically significant.

Silencing experiments were conducted for 48 (Fig. 5a-d) and 72 hours (Fig. 5e-h) with PEX1siRNA in NRVFs. PEX1 mRNA levels were reduced $31 \%$ at $48 \mathrm{~h}(\mathrm{P}<0.05)$ and $37 \%$ at $72 \mathrm{~h}(\mathrm{P}<0.005)$ compared to control (Fig. 5a, e). Significant changes in gene expression were specifically observed after 72 hours (Fig. 5b): IL-6 and FN-1 mRNA levels were diminished (29\% and 17\%, P<0.01; Fig. $5 f, h)$, and a similar decreasing trend (34\%, not significant) was noted for MMP-9 (Fig. $5 g$ ).

\section{PEX1 activates the MMP-9 promoter}

Both in vivo and in vitro results suggest that PEX1 may directly regulate pro-fibrotic genes, including MMP-9. As shown in Fig. 6, also protein levels increased subsequent to PEX1 overexpression in vivo already at 3 days. The promoter region of MMP-9 has a PEX1 binding region (PERE), upstream 
of the coding region (Fig. 7a) which is conserved in the promoters of human, mouse, and rat genomes. Therefore, we used a luciferase reporter approach to explore whether PEX1 could directly bind the promoter region of MMP-9 and regulate MMP-9 transcription. COS-1 cells were transfected with pCMV-PEX1 and PGL-III-pMMP9-Luc plasmids. In reporter gene studies, PEX1 increased MMP-9 promoter activity in a dose-dependent manner (Fig. 7b), demonstrating that PEX1 activates the promoter of MMP-9.

\section{PEX1 overexpression leads to reactivation of the fetal gene program}

Next, we studied the effects of gain- and loss-of PEX1 function on transcriptional regulation of fetal gene program in vivo. Gene expression analysis by RT-qPCR revealed activation of genes associated with the hypertrophic process in response to PEX1 overexpression, while the antisense construct had opposing effects. Significant increases of the mRNA levels of A-type natriuretic peptide (ANP), BNP and skeletal $\alpha$-actin (Acta1) (Electronic Supplementary Material, Fig. S2a, b, g) were detected at day 3 of PEX1 overexpression, while PEX1 silencing significantly decreased BNP, Acta1, and cardiac $\alpha$-actin (Actc1) mRNA levels (Electronic Supplementary Material, Fig. S2). In addition, significantly reduced $\alpha-\mathrm{MHC}$ to $\beta-\mathrm{MHC}$ and Actc1 to Acta1 ratios were detected in PEX1 injected hearts when compared to the LacZ control group (Electronic Supplementary Material, Fig. S2e, h), indicating the transcriptional switch from adult to fetal isoforms of contractile genes.

\section{Effects of gain- and loss-of PEX1 on cardiac function and structure in adult heart}

The cardiac function and structure were evaluated by echocardiography in vivo. Overall, the results of echocardiography analysis show that augmentation or silencing of PEX1 expression had no major effect on LV structure and function in normal rat hearts during the 2 weeks follow-up period. Yet, in agreement with the decreased fibrotic process, AsPEX1 adenoviral delivery slightly but significantly increased LVEF and LVFS at 2 weeks (Table1). As the LV remodeling process is characterized by the increased presence of apoptotic and stem cells, increased cell proliferation, and hypertrophied myocytes, we also analyzed these parameters by immunological detection in LV histological cross 
sections. As shown in Electronic Supplementary Material, Fig. S3, no significant changes in number of apoptotic cells, Ki-67+ cells, c-kit+ cells or myocyte size were observed.

\section{Effects of PEX1 overexpression and silencing in Ang II-induced hypertension and post- infarction in adult heart}

Finally, the effects of PEX1 and AsPEX1 gene transfers on myocardial fibrosis were examined post$\mathrm{MI}$ and in Ang II-induced hypertension in adult rats by quantification of expression of fibrotic genes levels and staining of histological sections by Masson's trichrome. Adenovirus-mediated PEX1 gene delivery increased PEX1 mRNA levels in both experimental models at 1 week while the downregulation of PEX gene expression by Ad5-AsPEX1 local injection was not statistically significant (Fig. 8a, b). There was a tendency towards augmented fibrosis by overexpressing PEX1, but this change was not statistically significant (Fig. 8g, h). In Ang Il-induced hypertension, PEX1 overexpression promoted the expression of the fibrotic genes, including collagen la1 and IIla1 isoforms, FN-1 and MMP-2. Although TGF $\beta 1$ levels were down-regulated at 1 week by the antisense construct, surprisingly, the expression of MF marker $\alpha$-SMA was up-regulated by PEX1 overexpression in Ang II-induced hypertension model (Fig. 8a). PEX1 adenovirus-mediated overexpression post-infarction caused significant increase of IL-6 expression and down-regulation of a-SMA expression at 1 week. Gene expression analysis of the animals subjected to LAD in combination with local adenoviral injection of AsPEX1 revealed significant down-regulation of CTGF, FN-1 and TGFß1 at 1 week (Fig. 8b) and of TGFß1 and MMP-2 at 2 weeks (data not shown).

\section{Discussion}

The heart responds to biomechanical stress and neurohumoral stimuli by activating signaling pathways that induce myocardial remodeling. Cardiac fibroblasts play an essential role in the remodeling response, as they are not only important modulators of ECM homeostasis but also of the inflammatory response, acting as source of matricellular components, cytokines and growth factors (Lindner et al. 2014). ECM production and crosslinking are keys to provide a support structure during the healing process. However, loss of homeostatic balance of ECM remodeling and excessive 
accumulation of structural proteins, such as collagens, leads to stiffer myocardial tissue, loss of contractility and eventually heart failure. PEX1 is a transcriptional regulator of the hypertrophic signaling, which is activated by a1-adrenergic stimulus, and co-operates with GATA4 to induce cardiac hypertrophic response (Debrus et al. 2005; Komati et al. 2011). The current study shows that PEX1, in addition to regulating cardiomyocyte hypertrophy, influences ECM turnover and cardiac fibrosis in the adult heart.

The main finding of this study is that PEX1 regulates key pro-fibrotic genes in the heart. In our study, cardiac fibrosis was increased by local PEX1 injection into the LV wall of normal adult rat hearts. Consistent with the increased fibrosis, expression of pro-fibrotic factors was activated subsequently to overexpression of PEX1. Accordingly, silencing of PEX1 repressed the expression of these pro-fibrotic genes. Particularly, we observed significant changes in the expression of cytokines, matrix metalloproteases and matricellular proteins, which all play a role in the inflammatory and fibrotic responses to pathological stress. In addition, overexpression of PEX1 in LV of adult rats up-regulated cardiac hypertrophy associated genes, noted by activation of ANP and BNP gene expression, and reactivation of the fetal gene program, which all are fundamental features of hypertrophic response of cardiomyocytes (Chien 1999; Oka et al. 2007; Braunwald 2013). These latter results are in line with the previous study, where PEX1 overexpression in cardiomyocytes was sufficient to induce myocardial hypertrophy and reactivation of the fetal gene program (Komati et al. 2011). However, Komati and colleagues (Komati et al. 2011) did not find evidence of ECM remodeling by overexpressing PEX1 in cardiac myocytes. These differences observed in vivo experiments are most likely due to the different experimental models used. In the study by Komati et al. (Komati et al. 2011), a conditional transgenic mouse line that overexpresses PEX1 specifically and intensively in cardiomyocytes upon tamoxifen administration was used, whereas in our study PEX1 overexpression was more transient and PEX1 co-localized with $\alpha$-actinin and $\mathrm{P} 4 \mathrm{H}$, indicating that PEX1 overexpression occurred in both myocytes and fibroblasts.

TGF $\beta 1$ is a major contributor of the profibrotic response, and also several cytokines such as IL-6 play a relevant role in the early response to myocardial stress, and the development of fibrosis. In the present study, TGF $\beta 1$ expression in adult rat heart was induced by PEX1 overexpression and 
inhibited upon silencing, whereas IL-6 was up-regulated by PEX1 consistently in all our experimental models. Once activated, TGF $\beta 1$ can elicit fibroblast to MF transdifferentiation by inducing the expression of collagens (specially I and III isoforms), FN-1, and $\alpha$-SMA (Yu and Stamenkovic 2000; Dobaczewski et al. 2010). Of note, in view of the role of $\alpha$-SMA in transdifferentiation, it localized with PEX1 in LV 3 days after gene delivery. Furthermore, TGF $\beta$ promotes synthesis of matricellular and ECM proteins, such as fibronectin, CTGF, and IL-6, which in turn regulate the capability of TGF $\beta$ to induce MF transdifferentiation (Serini et al. 1998; Chen et al. 2000; Ma et al. 2012). Independently of the etiology of the stimuli, MF transdifferentiation is a hallmark of both reactive and reparative fibrosis (Cleutjens et al. 1995; Sun 2000). Moreover, IL-6 contributes to the inflammatory response in heart (Gullestad et al. 2012; Turner 2015) and several studies provide evidence of IL-6 as a mediator in cardiac fibrosis (Sano et al. 2000; Meléndez et al. 2010; Ma et al. 2012). Meléndez and colleagues showed that IL-6 administration could induce fibrosis, concentric LV hypertrophy and diastolic dysfunction, consistent with the response occurring in hypertensive hearts (Meléndez et al. 2010). Our gene expression analysis revealed that mRNA levels of pro-fibrotic genes collagens la1 and IIla1, FN-1, CTGF, and a-SMA were increased by PEX1 overexpression in normal hearts in vivo similarly to TGF $\beta 1$. Altogether this is in agreement with the activation of TGF $\beta 1$ fibrotic pathway subsequent to PEX1 overexpression, shown by increased presence downstream targets linked to MF transdifferentiation and upstream modulators of TGF $\beta 1$. Overall, these findings provide evidence for the fibroblast activation as the main factor responsible for the cardiac fibrosis subsequently to PEX1 overexpression. Yet, despite that PEX1 did not increase the number of apoptotic nuclei in our in vivo experimental model, we cannot exclude the possibility of other cell death processes, such as necrosis, contributed to the observed increase in cardiac fibrosis. It is also noteworthy that PEX1 gain- and loss-of-function experiments in vitro in rat neonatal cardiac fibroblasts and myocytes revealed that pro-fibrotic gene expression was affected by PEX1 predominantly in fibroblasts.

The latent form of TGF $\beta 1$ can be produced by a number of cell types including the main resident cells of the heart, fibroblasts and myocytes; yet inflammatory cells, such as neutrophils and macrophages, which can infiltrate the myocardium, constitute another important source. Proteases MMP-2 and MMP-9 have been shown to release the ECM-bound latent TGF $\beta 1$ (Yu and Stamenkovic 
2000; Bujak and Frangogiannis 2007; Dayer and Stamenkovic 2015). Interestingly, MMP-2 and MMP-9 were markedly up-regulated in vivo and in cultured NRVF. More importantly, PEX1 has stimulatory effect on MMP-9 protein levels in vivo and MMP-9 promoter activity, as shown by luciferase reporter gene experiments. Therefore, it is tempting to speculate that PEX1 possibly modulates ECM homeostasis by increasing the amount of MMP-9 produced by the cells, leading to increasing amounts of active MMP-9, which then could release and activate interstitial TGF $\beta 1$ latent form. MMP-9 is an active player in the inflammatory-fibrotic response secreted by a wide number of cell types, from endothelial to neutrophils, macrophages and fibroblasts (Yabluchanskiy et al. 2013; Ma et al. 2014). Thus, the increased MMP-9 expression in vivo may be induced in inflammatory cells as well, possibly caused by PEX1 promotion of the inflammatory response, as indicated by increased expression of inflammatory markers in response to PEX1 overexpression. MMP-9 is strongly present in cardiovascular diseases and associated with negative outcome, such as increased aortic stiffness and adverse LVH in hypertension or post-MI LV dilatation, and several studies have shown the benefits of inhibiting or silencing MMP-9 (Romanic et al. 2001; Ikonomidis et al. 2005; Takai et al. 2007; Krishnamurthy et al. 2009).

Activation of $\alpha_{1}$-adrenergic receptors in animal models of hypertension in vivo and in cultured cardiomyocytes up-regulates PEX1 expression (Debrus et al. 2005; Komati et al. 2011). Therefore, we explored the effects of PEX1 modulation by local adenoviral gene transfer in Ang II-mediated hypertension and post-infarction. Even though PEX1 mRNA was significantly overexpressed, we did not detect statistically significant changes in the fibrotic tissue content. Moreover, PEX1 effect on $\alpha-$ SMA expression was opposite to the observations in normal hearts. In line with the findings in pathological models, a strong down-regulation of $\alpha$-SMA and CTGF mRNAs in response to PEX1 overexpression was detected also in NRVF primary cultures. One possible explanation to weak induction of fibrosis-associated genes in diseased hearts is that PEX1 levels are elevated in Ang IIinduced hypertension (Jurado Acosta et al., unpublished data) or post-infarction. On the other hand, TGF $\beta 1$ expression was down-regulated by PEX1 silencing in the models of hypertension and myocardial infarction, indicating that PEX1 may contribute to up-regulation of TGF $\beta 1$ expression in response to stress stimuli in the heart. TGF $\beta 1$-induced MF contractile capabilities require of CTGF 
(Garrett et al. 2004), yet incorporation of $\alpha$-SMA into the fibroblast cytoskeleton confers contractile properties, the most defining characteristic of MF (Ma et al. 2014). However, although PEX1 affects the fibrotic process in heart, the cardiac function was preserved in our experimental models, similarly to previous studies where transgenic mice with inducible PEX1 developed LVH without effect on the heart function (Komati et al. 2011). In a pathological environment, other neurohumoral factors, physical stimuli and damage-associated molecules are present which may alter or complement PEX1 actions observed in a normal heart. A limitation of this study is that the in vitro models are isolated cells in a much simpler context than the ones found in vivo, lacking ECM, paracrine signaling and communication between different cell types. In addition, the interpretation of the findings in models of angiotensin-induced cardiac remodeling and myocardial infarction is challenging, since the changes in endogenous levels of PEX1 following cardiac stress injuries complicate interpretation of any effects of the overexpression and knockdown strategies. Nevertheless, our study clearly showed that PEX1 promotes the expression of MMPs in NRVF, even though the effects of the proteolytic activity observed in vivo may be lost without ECM, since there is no interstitial TGF $\beta$ that cannot be activated to trigger the fibrotic signaling. The precise mechanisms for the distinct cardiac actions of PEX1 remain to be established, but may be due to diverse pathophysiological processes occurring in MI and hypertension-induced heart disease (Jessup and Brozena 2003; McMurray and Pfeffer 2005). Previously we have reported that several gene transfers in these animal models lead to different effects on cardiac structure and function depending on the distinct signaling networks activated in each experimental model (Tenhunen et al. 2006a; Tenhunen et al. 2006b; Moilanen et al. 2011). Overall, in the present study we could study structural and functional changes during the early remodeling process, because adenoviral gene delivery results in transient increase in PEX1 expression. Therefore, to study the longer-term effects on extracellular matrix turnover, detailed experiments in other models are needed.

In conclusion, our findings provide a new insight into the regulation of ECM remodeling and the fibrotic process in the heart. The results indicate that PEX1 induces ECM remodeling and a beneficial type of fibrosis, which imitates the protective fibrotic response occurring in stressed myocardium. Interestingly, very recently adult fibroblasts reprogramming have been used to 
generate proliferative induced cardiac progenitor cells which could be directed into different cardiovascular cell lineages (Lalit et al. 2016), highlighting the ability of fibroblasts to protect and regenerate the heart. Our results further indicate that PEX1 stimulates MMP-9 promoter activity, which might trigger the pro-fibrotic response directed by PEX1. The schematic diagram in Fig.9 illustrates a possible mechanism of action according to the findings of this study combined with previous findings. We suggest that through promoting MMP-9 expression, PEX1 induces the activation of interstitial TGF $\beta 1$, which in turn activates the expression of ECM components by inducing the transdifferentiation into a synthetic and contractile cell type, myofibroblasts. In addition to MMPs effects on ECM turnover, processing of cytokines, chemokines and growth factors, there are other ways by which MMPs can influence inflammatory and fibrotic pathways. In this study, PEX1 overexpression in non-myocyte resident cells, such as fibroblasts, was predominantly responsible for the fibrosis-associated changes observed.

\section{Supplementary Material}

Electronic Supplementary Material is available at Cell and Tissue Research online.

\section{Acknowledgements}

We thank Professor Wolfgang Eberhardt (Pharmazentrum Frankfurt/ZAFES, Klinikum der GoetheUniversität Frankfurt, Frankfurt am Main, Germany) for the pGL-III-pMMP9-Luc construct and Professor Olli Vuolteenaho (Research Unit of Biomedicine, University of Oulu, Finland) for kindly providing Ang II. We also thank Marja Arbelius, Kati Lampinen, Kirsi Salo, and Sirpa Rutanen for their expert technical assistance.

Conflict of Interest: The authors declare that they have no conflict of interest 


\section{References}

Abreu JG, Ketpura NI, Reversade B, De Robertis EM (2002) Connective-tissue growth factor (CTGF) modulates cell signalling by BMP and TGF-beta. Nat Cell Biol 4:599-604

Braunwald E (2013) Heart failure. JACC Heart Fail 1:1-20

Bujak M, Frangogiannis NG (2007) The role of TGF-beta signaling in myocardial infarction and cardiac remodeling. Cardiovasc Res 74:184-95

Chan W, Duffy SJ, White DA, Gao X-M, Du X-J, Ellims AH, Dart AM, Taylor AJ (2012) Acute left ventricular remodeling following myocardial infarction: coupling of regional healing with remote extracellular matrix expansion. JACC Cardiovasc Imaging 5:884-93

Chen MM, Lam A, Abraham JA, Schreiner GF, Joly AH (2000) CTGF Expression is Induced by TGFB in Cardiac Fibroblasts and Cardiac Myocytes: a Potential Role in Heart Fibrosis. J Mol Cell Cardiol 32:1805-19

Chien KR (1999) Stress pathways and heart failure. Cell 98:555-558

Cleutjens JP, Verluyten MJ, Smiths JF, Daemen MJ (1995) Collagen remodeling after myocardial infarction in the rat heart. Am J Pathol 147:325-38

Dayer C, Stamenkovic I (2015) Recruitment of Matrix Metalloproteinase-9 (MMP-9) to the Fibroblast Cell Surface by Lysyl Hydroxylase 3 (LH3) Triggers Transforming Growth Factor- $\beta$ (TGF- $\beta$ ) Activation and Fibroblast Differentiation. J Biol Chem 290:13763-78

Debrus S, Rahbani L, Marttila M, Delorme B, Paradis P, Nemer M (2005) The zinc finger-only protein Zfp260 is a novel cardiac regulator and a nuclear effector of $\alpha 1$-adrenergic signaling. Mol Cell Biol 25:8669-8682

Dobaczewski M, Gonzalez-Quesada C, Frangogiannis NG (2010) The extracellular matrix as a modulator of the inflammatory and reparative response following myocardial infarction. $\mathrm{J}$ Mol Cell Cardiol 48:504-11

Eberhardt W, Akool E-S, Rebhan J, Frank S, Beck K-F, Franzen R, Hamada FMA, Pfeilschifter J (2002) Inhibition of cytokine-induced matrix metalloproteinase 9 expression by peroxisome proliferator-activated receptor alpha agonists is indirect and due to a NO-mediated reduction of mRNA stability. J Biol Chem 277:33518-28 
Fan D, Takawale A, Lee J, Kassiri Z (2012) Cardiac fibroblasts, fibrosis and extracellular matrix remodeling in heart disease. Fibrogenesis Tissue Repair 5:15

Frangogiannis N (2000) Myofibroblasts in reperfused myocardial infarcts express the embryonic form of smooth muscle myosin heavy chain (SMemb). Cardiovasc Res 48:89-100

Garrett Q, Khaw PT, Blalock TD, Schultz GS, Grotendorst GR, Daniels JT (2004) Involvement of

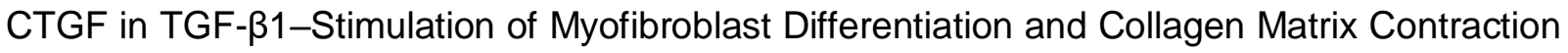
in the Presence of Mechanical Stress. Investig Opthalmology Vis Sci 45:1109-16

Gullestad L, Ueland T, Vinge LE, Finsen A, Yndestad A, Aukrust P (2012) Inflammatory cytokines in heart failure: mediators and markers. Cardiology 122:23-35

Ikonomidis JS, Hendrick JW, Parkhurst AM, Herron AR, Escobar PG, Dowdy KB, Stroud RE, Hapke E, Zile MR, Spinale FG (2005) Accelerated LV remodeling after myocardial infarction in TIMP. 1-deficient mice: effects of exogenous MMP inhibition. Am J Physiol Heart Circ Physiol 288:H149-58

Jessup M, Brozena S (2003) Heart Failure. N Engl J Med 348:2007-2018

Komati H, Maharsy W, Beauregard J, Hayek S, Nemer M (2011) ZFP260 is an inducer of cardiac hypertrophy and a nuclear mediator of endothelin-1 signaling. J Biol Chem 286:1508-16

Krishnamurthy P, Peterson JT, Subramanian V, Singh M, Singh K (2009) Inhibition of matrix metalloproteinases improves left ventricular function in mice lacking osteopontin after myocardial infarction. Mol Cell Biochem 322:53-62

Lalit PA, Salick MR, Nelson DO, Squirrell JM, Shafer CM, Patel NG, Saeed I, Schmuck EG, Markandeya YS, Wong R, Lea MR, Eliceiri KW, Hacker TA, Crone WC, Kyba M, Garry DJ, Stewart R, Thomson JA, Downs KM, Lyons GE, Kamp TJ (2016) Lineage Reprogramming of Fibroblasts into Proliferative Induced Cardiac Progenitor Cells by Defined Factors. Cell Stem Cell 18:354-367

Leask A, Abraham DJ (2004) TGF-beta signaling and the fibrotic response. FASEB J 18:816-27

Lindner D, Zietsch C, Tank J, Sossalla S, Fluschnik N, Hinrichs S, Maier L, Poller W, Blankenberg S, Schultheiss H-P, Tschöpe C, Westermann D (2014) Cardiac fibroblasts support cardiac inflammation in heart failure. Basic Res Cardiol 109:428 
Ma F, Li Y, Jia L, Han Y, Cheng J, Li H, Qi Y, Du J (2012) Macrophage-stimulated cardiac fibroblast production of IL-6 is essential for TGF $\beta /$ Smad activation and cardiac fibrosis induced by angiotensin II. PLoS One 7:e35144

Ma Y, de Castro Brás LE, Toba H, lyer RP, Hall ME, Winniford MD, Lange RA, Tyagi SC, Lindsey ML (2014) Myofibroblasts and the extracellular matrix network in post-myocardial infarction cardiac remodeling. Pflügers Arch Eur J Physiol 466:1113-27

McMurray JJ, Pfeffer MA (2005) Heart failure. Lancet 365:1877-1889

Meléndez GC, McLarty JL, Levick SP, Du Y, Janicki JS, Brower GL (2010) Interleukin 6 mediates myocardial fibrosis, concentric hypertrophy, and diastolic dysfunction in rats. Hypertension $56: 225-31$

Moilanen AM, Rysä J, Mustonen E, Serpi R, Aro J, Tokola H, Leskinen H, Manninen A, Levijoki J, Vuolteenaho O, Ruskoaho H (2011) Intramyocardial BNP gene delivery improves cardiac function through distinct context-dependent mechanisms. Circ Hear Fail 4:483-495

Oka T, Xu J, Molkentin JD (2007) Re-employment of developmental transcription factors in adult heart disease. Semin Cell Dev Biol 18:117-131

Pikkarainen S, Tokola H, Kerkelä R, Ruskoaho H (2004) GATA transcription factors in the developing and adult heart. Cardiovasc Res 63:196-207

Pikkarainen S, Tokola H, Majalahti-Palviainen T, Kerkela R, Hautala N, Bhalla SS, Charron F, Nemer M, Vuolteenaho O, Ruskoaho H (2003) GATA-4 is a nuclear mediator of mechanical stretchactivated hypertrophic program. J Biol Chem 278:23807-16

Romanic AM, Burns-Kurtis CL, Gout B, Berrebi-Bertrand I, Ohlstein EH (2001) Matrix metalloproteinase expression in cardiac myocytes following myocardial infarction in the rabbit. Life Sci 68:799-814

Rysä J, Tenhunen O, Serpi R, Soini Y, Nemer M, Leskinen H, Ruskoaho H (2010) GATA-4 is an angiogenic survival factor of the infarcted heart. Circ Heart Fail 3:440-50

Sano M, Fukuda K, Kodama H, Pan J, Saito M, Matsuzaki J, Takahashi T, Makino S, Kato T, Ogawa S (2000) Interleukin-6 family of cytokines mediate angiotensin II-induced cardiac hypertrophy in rodent cardiomyocytes. J Biol Chem 275:29717-23 
Serini G, Bochaton-Piallat ML, Ropraz P, Geinoz A, Borsi L, Zardi L, Gabbiani G (1998) The fibronectin domain ED-A is crucial for myofibroblastic phenotype induction by transforming growth factor- $\beta 1$. J Cell Biol 142:873-881

Serpi R, Tolonen A-M, Huusko J, Rysä J, Tenhunen O, Ylä-Herttuala S, Ruskoaho H (2011) Vascular endothelial growth factor-B gene transfer prevents angiotensin II-induced diastolic dysfunction via proliferation and capillary dilatation in rats. Cardiovasc Res 89:204-13

Shinde A V, Frangogiannis NG (2014) Fibroblasts in myocardial infarction: a role in inflammation and repair. J Mol Cell Cardiol 70:74-82

Sun Y (2000) Infarct scar: a dynamic tissue. Cardiovasc Res 46:250-256

Sun Y, Zhang JQ, Zhang JQ, Lamparter S (2000) Cardiac remodeling by fibrous tissue after infarction in rats. J Lab Clin Med 135:316-23

Suo M, Hautala N, Foldes G, Szokodi I, Toth M, Leskinen H, Uusimaa P, Vuolteenaho O, Nemer M, Ruskoaho H (2002) Posttranscriptional Control of BNP Gene Expression in Angiotensin IIInduced Hypertension. Hypertension 39:803-808

Takai S, Jin D, Inagaki S, Yamamoto D, Tanaka K, Miyazaki M (2007) Significance of matrix metalloproteinase-9 in cardiac dysfunction during the very acute phase after myocardial infarction in hamsters. Eur J Pharmacol 572:57-60

Talman V, Ruskoaho H (2016) Cardiac fibrosis in myocardial infarction---from repair and remodeling to regeneration. Cell Tissue Res 1-19 Jun 21. [Epub ahead of print] DOI: 10.1007/s00441-016$2431-9$

Tenhunen O, Rysä J, Ilves M, Soini Y, Ruskoaho H, Leskinen H (2006a) Identification of cell cycle regulatory and inflammatory genes as predominant targets of p38 mitogen-activated protein kinase in the heart. Circ Res 99:485-493

Tenhunen O, Soini Y, Ilves M, Rysä J, Tuukkanen J, Serpi R, Pennanen H, Ruskoaho H, Leskinen H (2006b) p38 Kinase rescues failing myocardium after myocardial infarction: evidence for angiogenic and anti-apoptotic mechanisms. FASEB J 20:1907-9

Turner NA (2015) Inflammatory and fibrotic responses of cardiac fibroblasts to myocardial damage associated molecular patterns (DAMPs). J Mol Cell Cardiol 94:189-200 
van Nieuwenhoven FA, Turner NA (2013) The role of cardiac fibroblasts in the transition from inflammation to fibrosis following myocardial infarction. Vascul Pharmacol 58:182-188.

Yabluchanskiy A, Ma Y, lyer RP, Hall ME, Lindsey ML (2013) Matrix metalloproteinase-9: Many shades of function in cardiovascular disease. Physiology (Bethesda) 28:391-403

Yu Q, Stamenkovic I (2000) Cell surface-localized matrix metalloproteinase-9 proteolytically activates TGF-beta and promotes tumor invasion and angiogenesis. Genes \& Dev 14:163-176 


\section{Figure Legends}

Fig. 1 Cardiac specific augmentation and silencing of PEX1 expression by adenoviral gene delivery into the LV in rats in vivo. a PEX1 mRNA levels were measured by RT-qPCR from LV extracts and normalized to Glyceraldehyde 3-phosphate dehydrogenase (GAPDH) housekeeping gene. The results are expressed as mean $\pm S D\left(N=5\right.$ to 10 animals/group). ${ }^{*} \mathrm{P}<0.05,{ }^{*} \mathrm{P}<0.01$, ${ }^{* * *} \mathrm{P}<0.005$ versus LacZ control (Student's t-test or Mann Whitney U-test). b-d"' Efficiency of gene transfer was confirmed by immunohistochemical staining against PEX1. Representative images from LacZ (b-b"), HA-PEX1 (c-c") and AsPEX1 (d-d") adenovirus-treated hearts are shown. Hearts treated with PEX1 show strongest signal. The scale bar is $100 \mu \mathrm{m}$

Fig. 2 PEX1 adenoviral gene transfer in rats in vivo increases PEX1 levels in cardiac myocytes and fibroblasts. Cell specificity of PEX1 protein expression was evaluated by immunofluorescent co-staining with prolyl-4-hydroxylase $(\mathrm{P} 4 \mathrm{H}$, a fibroblast marker) and sarcomeric $\alpha$-actinin (a myocyte marker). Representative images are shown from LacZ (a-b'”), PEX1 (c-d'”) and AsPEX1 (e-f'"') treated hearts at day 3 after injection. Consecutive slices were used for $\alpha$-actinin and $\mathrm{P} 4 \mathrm{H}$ double stainings with PEX1, and images depict the same LV area for $\alpha$-actinin and P4H. White arrow heads indicate co-localization of the specific cell marker and PEX1 in the merged images (a"', b"', c"', d"', e"', f'”). The scale bar is $50 \mu \mathrm{m}$

Fig. 3 PEX1 overexpression in normal rat heart increases fibrosis and expression of genes involved in the fibrotic process. a-c" Representative images LV histological sections stained with Masson's trichrome (a, a', b, b', c, c') and picrosirius red under polarized light (a", b", c'). d Masson's trichrome fibrotic tissue stain quantification from 5 hotspots in the LV. The results are expressed as mean $\pm S D$. * $P<0.05$ versus LacZ control ( $N=6$ to 9 animals/group, Student's t-test). em Fibrotic gene program was activated in normal rat heart after PEX1 overexpression when compared to LacZ control, as evident by increased mRNA levels of collagens $(\mathbf{e}, \mathbf{f})$, cytokine IL-6 (g), MMP-9 and MMP-2 (h, i), FN-1 (j), TGF $\beta 1$ (k), CTGF (I), and $\alpha-S M A(\mathbf{m})$ at day 3 after injection. mRNA levels were measured by RT-qPCR from LV extracts and normalized to GAPDH 
housekeeping gene. The results are expressed as mean $\pm S D .{ }^{*} P<0.05,{ }^{* \star} P<0.01,{ }^{* \star *} P<0.005$ versus LacZ control ( $N=6$ to 9 animals/group, Student's t-test or Mann Whitney U-test)

Fig. 4 Differential effects of PEX1 adenoviral overexpression in NRVF and NRVM in vitro. The mRNA levels were quantified by RTqPCR 24 hours (a-g) or 48 hours (h-n) after adenovirus gene transfer and normalized to $18 \mathrm{~S}$ housekeeping gene. The results are expressed as mean $\pm S D$. * $\mathrm{P}<0.05,{ }^{* *} \mathrm{P}<0.01,{ }^{* *} \mathrm{P}<0.005$ versus LacZ control $(\mathrm{N}=5$ to $7(\mathbf{a}-\mathbf{g})$ and 2 to 3 (h-n), Student's t-test or Mann Whitney U-test)

Fig. 5 Effects of PEX1 silencing in NRVF. Primary cells were transfected with PEX1-siRNA or AllStar-siRNA for 48 hours (a-d) or 72 hours $(\mathbf{e}-\mathbf{h})$. The mRNA levels of PEX1 (a, e), IL-6 (b, f), MMP-9 (c, g) and FN-1 (d, h) were quantified by RT-qPCR. The results are expressed as mean $\pm S D{ }^{*} \mathrm{P}<0.05,{ }^{* *} \mathrm{P}<0.01,{ }^{* * *} \mathrm{P}<0.005$ versus AllStar-siRNA negative control ( $\mathrm{N}=5$ to 6 , Student's t-test or Mann Whitney U-test)

Fig. 6 MMP-9 protein levels were increased 3 days after PEX1 adenoviral gene transfer in normal heart. Quantification of MMP-9 nuclear and total protein levels. The MMP-9 (105kDa) values were normalized to LaminB (50kDa, nuclear protein fraction) or GAPDH (36kDa, total protein fraction), representative Western blots are shown. The results are expressed as mean $\pm S D{ }^{*} P<0.05$ relative to LacZ adenovirus control ( $N=3-4$, Student's t-test)

Fig. 7 PEX1 activates the MMP-9 promoter. a Sequence of the upstream $1.3 \mathrm{~kb}$ of the rat MMP-9 gene promoter. Putative binding region for PEX1, the phenylephrine response element binding site (PERE), is underlined. The schematic representation of the upstream region is included in the figure. $\mathbf{b}$ Luciferase activity of the MMP-9 promoter construct is increased in the presence of PEX1 expressing plasmid in a dose dependent manner. The results are expressed as mean $\pm S D$ * $\mathrm{P}<0.05,{ }^{* *} \mathrm{P}<0.01,{ }^{* * *} \mathrm{P}<0.005$ versus lower $\mathrm{PCGN}-\mathrm{PEX} 1$ concentration; $\wedge \mathrm{P}<0.05, \wedge \wedge \mathrm{P}<0.01$, ${ }^{\wedge \wedge \wedge} \mathrm{P}<0.005$ versus $\mathrm{pCGN}$ control $(\mathrm{N}=3$, Student's t-test)

Fig. 8 Effects of adenovirus mediated PEX1 gain-and loss-of-function in Ang II-induced hypertension and post-infarction in rats. Changes in fibrosis associated gene-expressions at 1 
week were quantified by RT-qPCR and normalized to GAPDH (a, b). Representative images of Masson's trichrome staining from LV histological sections from 1 week Ang II infused (c-d") and MI animals (e-f'). The scale bar is $50 \mu \mathrm{m}$. Fibrosis quantifications were made from 5 hotspots from the LV of Ang II-induced hypertension ( $\mathbf{g}$ ) and post-infarction (h) rats. The results are expressed as mean $\pm S D\left(N=6\right.$ to 9 animals/group). ${ }^{*} \mathrm{P}<0.05$, ${ }^{* *} \mathrm{P}<0.01,{ }^{* * *} \mathrm{P}<0.005$ versus LacZ control (Student's t-test or Mann Whitney U-test)

Fig. 9 A schematic presentation of the effects of PEX1 in the heart 
Figure 1

a
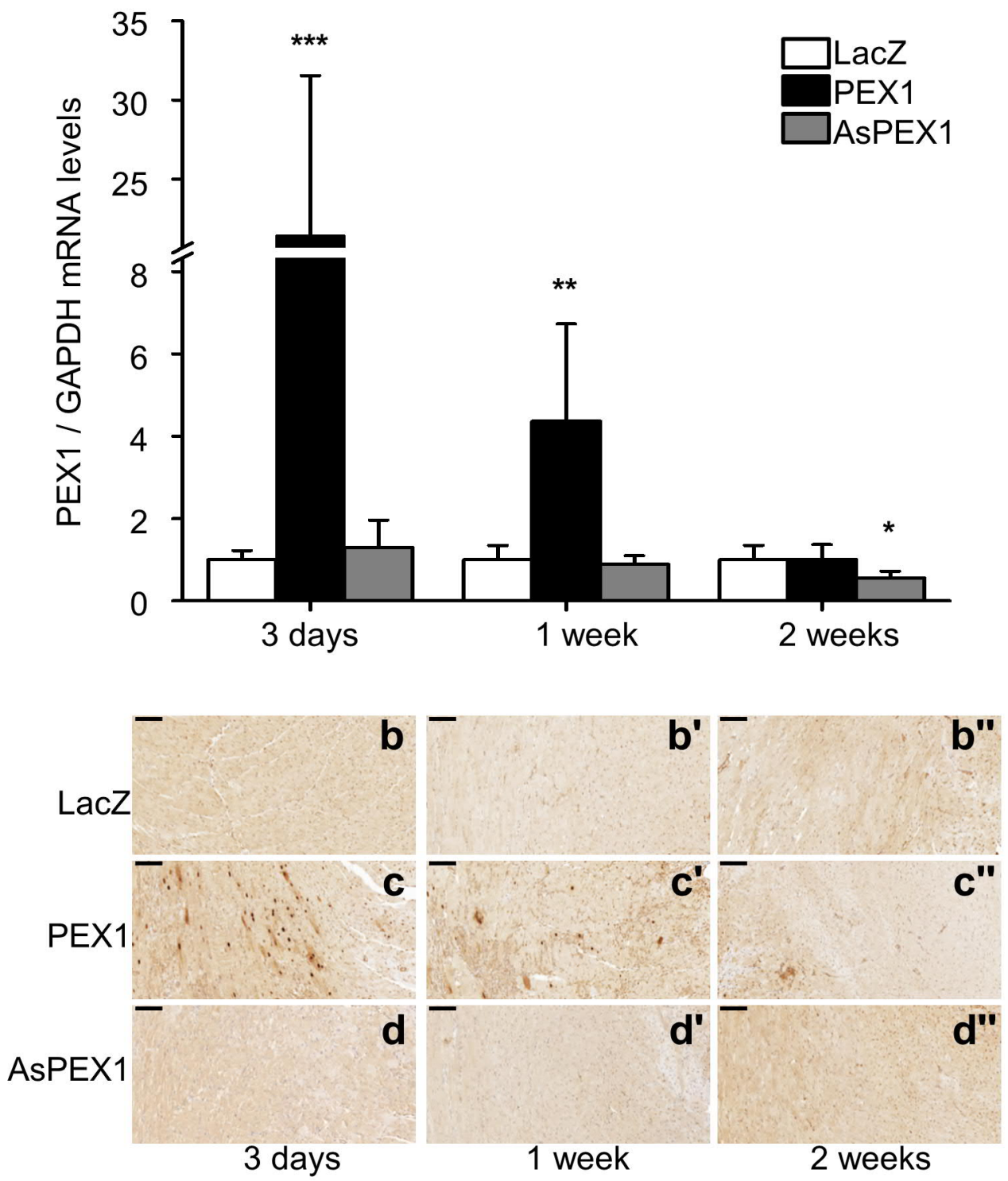
Figure 2

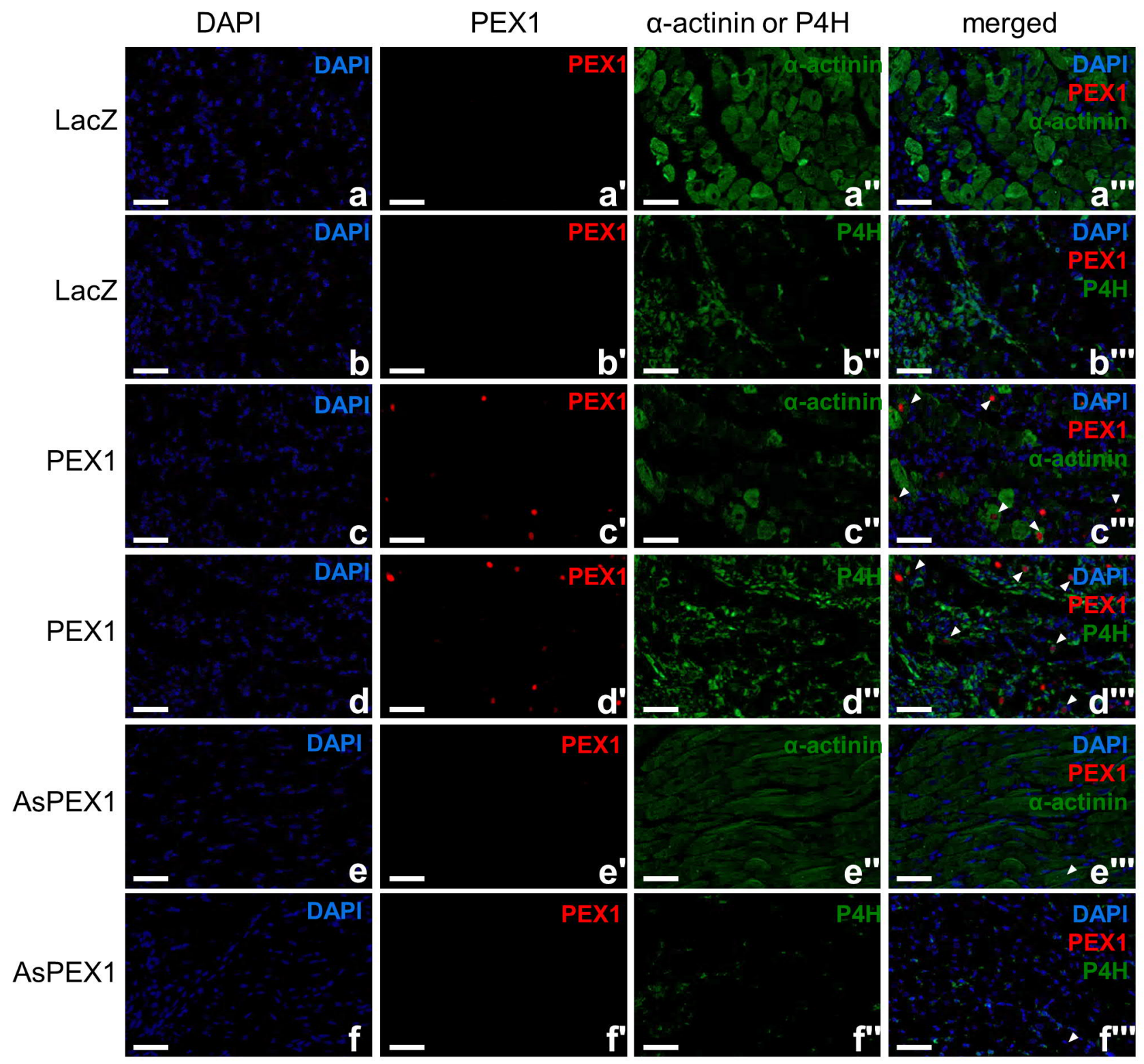


Figure 3
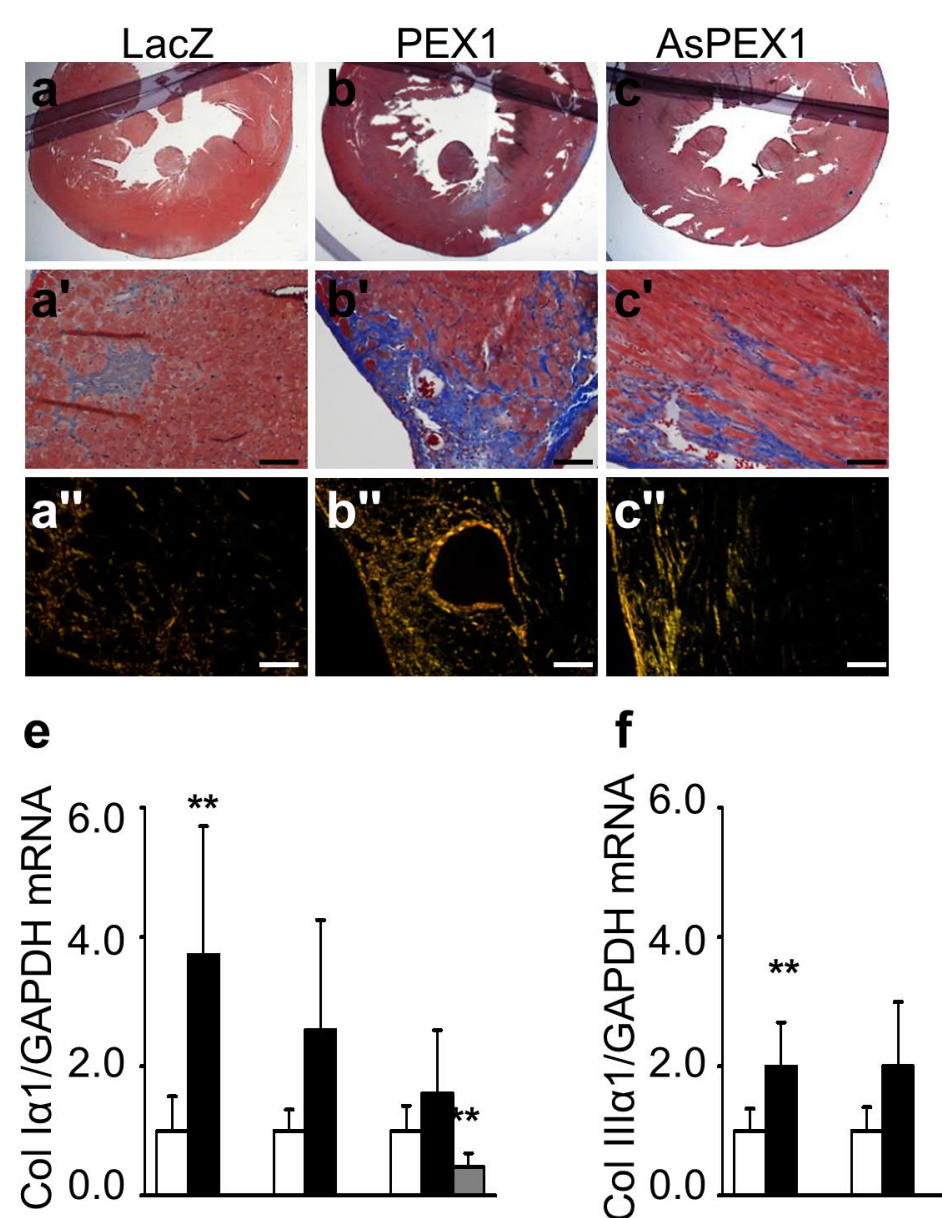

h

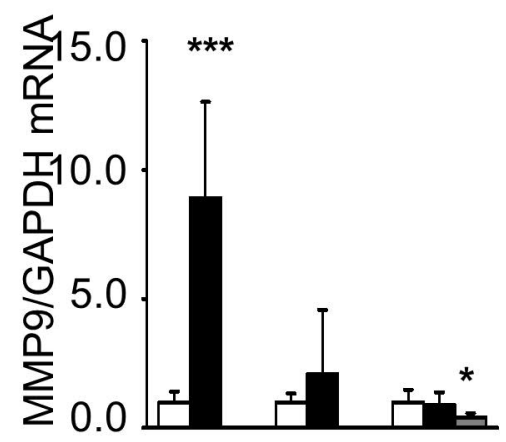

k

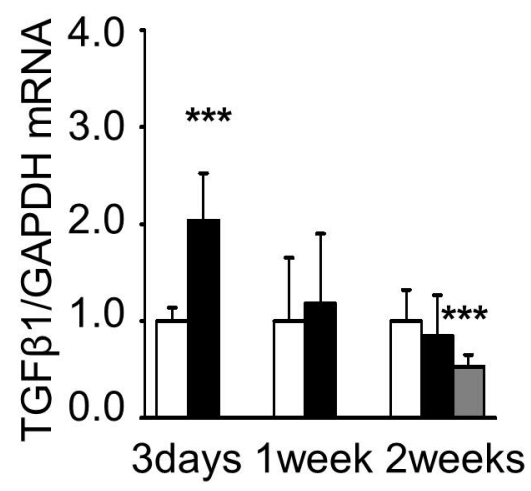

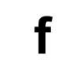
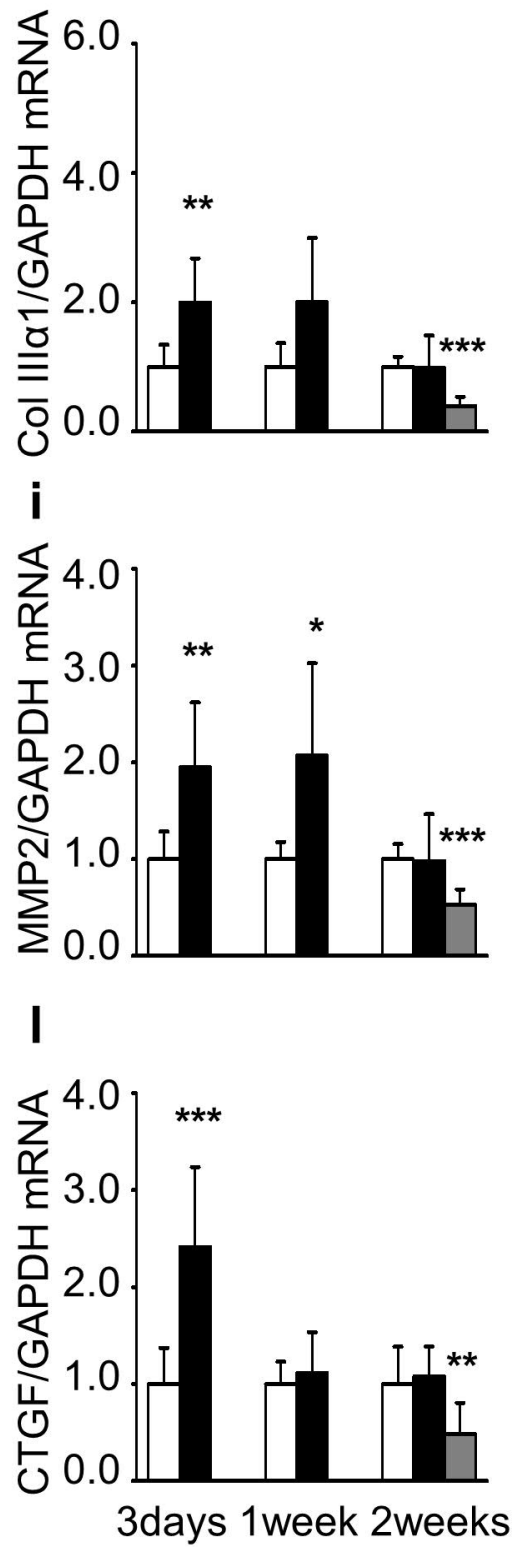

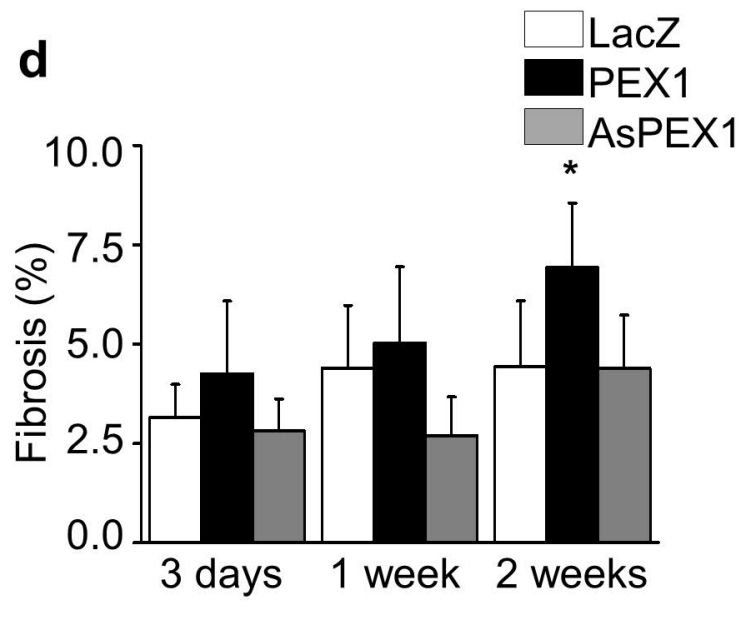

g
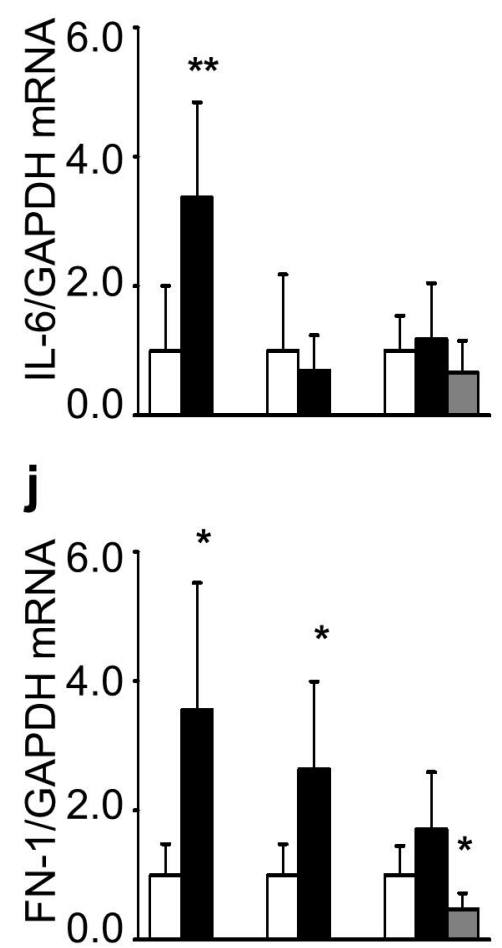

m

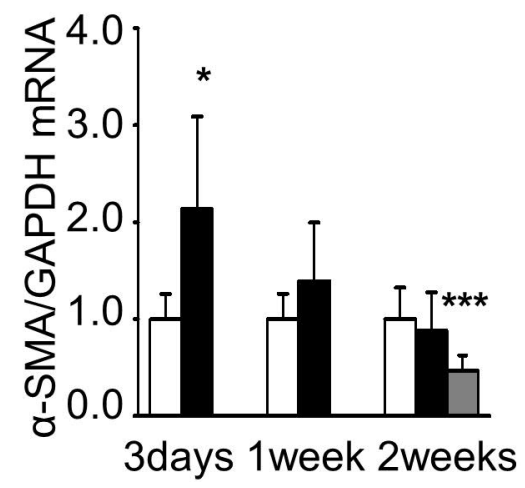


Figure 4
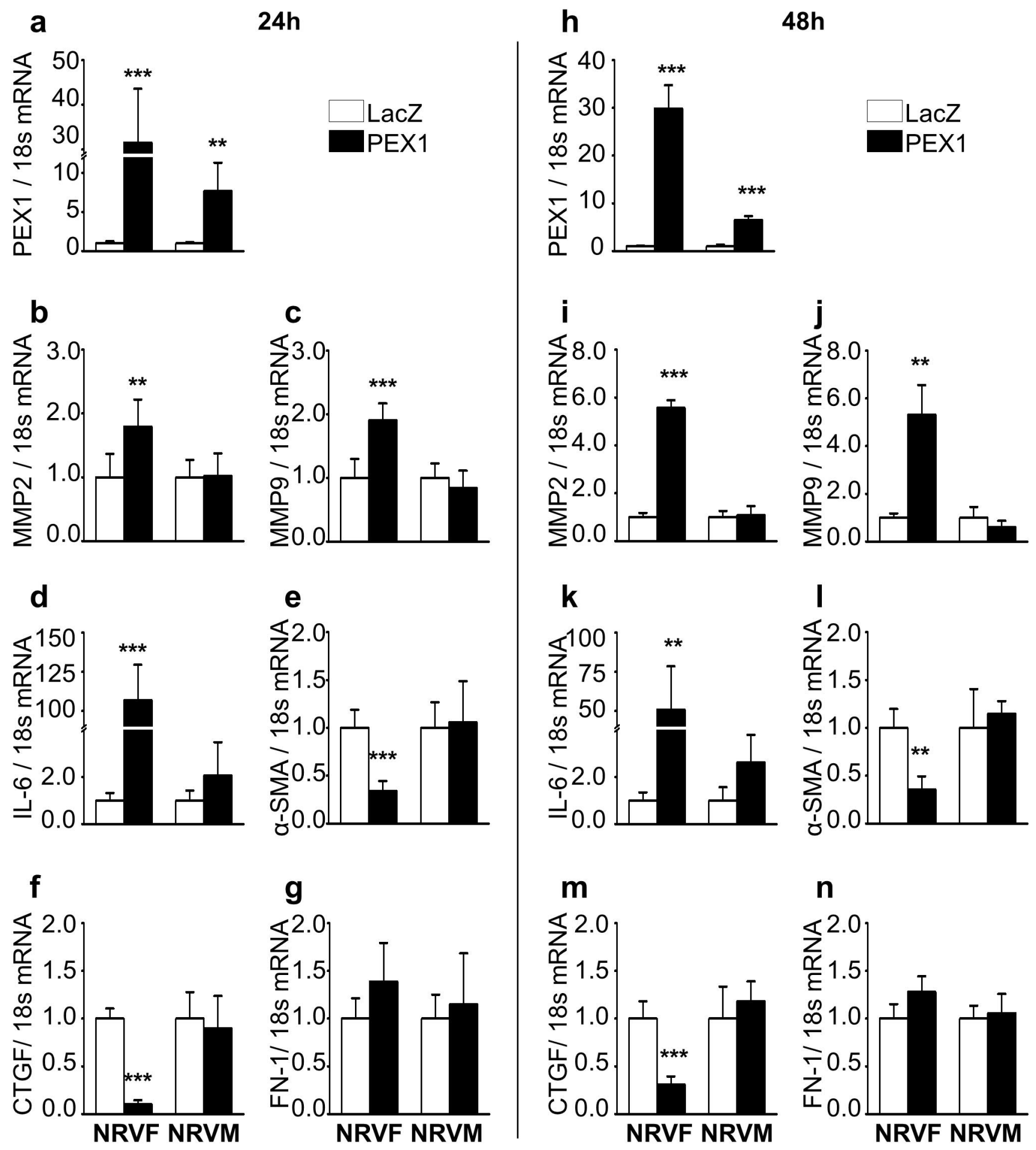

m

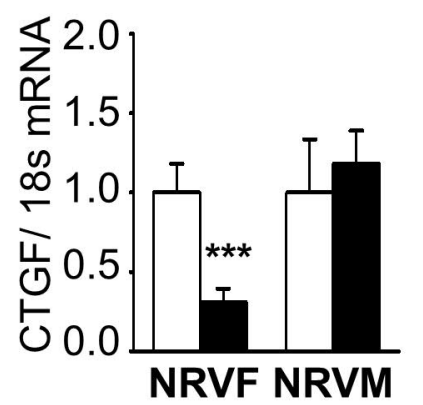

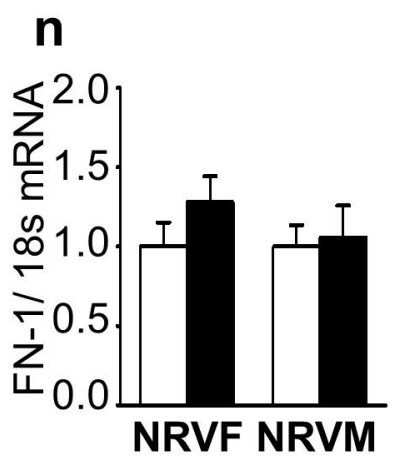


Figure 5
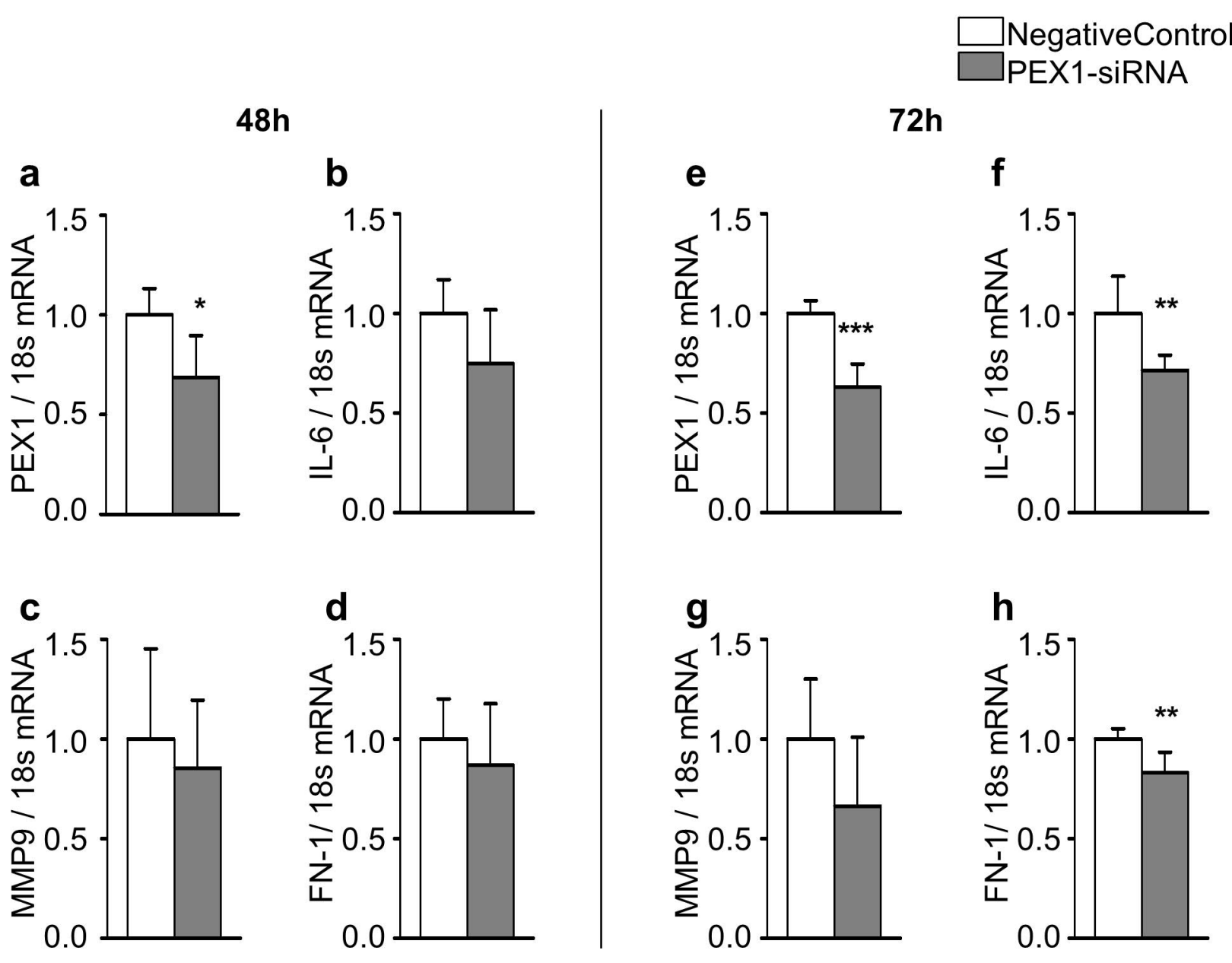
Figure 6

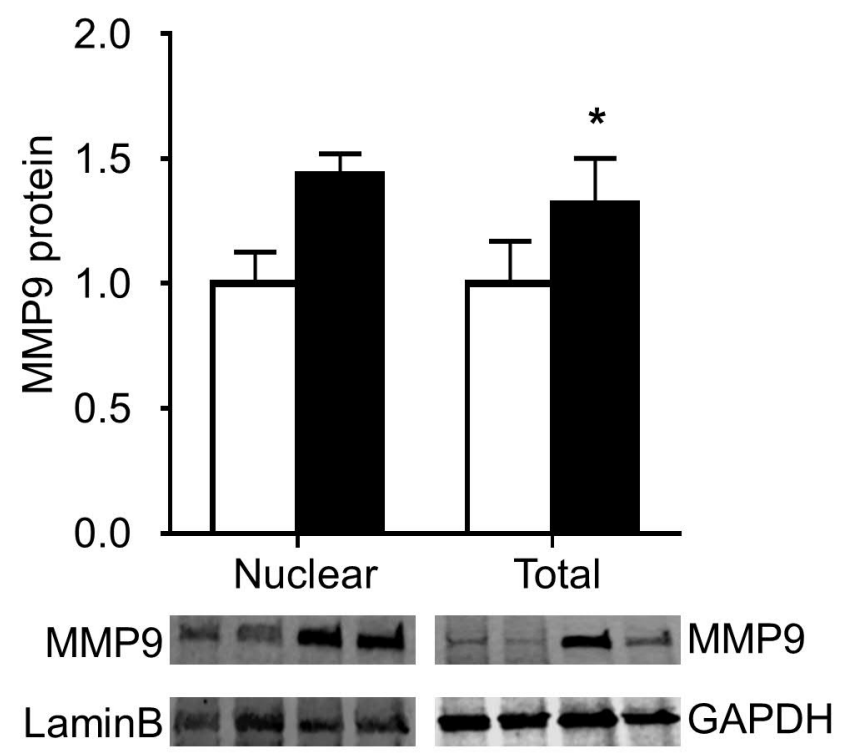


Figure 7

a

-1378 CTCACAGACTCATACGTCCCTTAGGAGATAGGATAGAGAGAGCACATCAT - 1328

-1327 AATGCTCAACACAATAAATCATAAAAAGTGAATTATCACTGTGGCTIGAAGG -1277

-1276 CGAAATGCTTGCCCAAGGTGACAAAGTGGGAAATGGTGAGGTGGGAA -1226

-1225 ATGGTGGACCCAGGACTGCAATTCAGTGCTGAAGACCATTGTCAGAAGCA -1175

-1174 TATAAGAGAAGCTGGGGAGCACGTCCAGCCTCCCCGCCCGTCTCACAGG -1124

-1123 TCTGTACATTAGGAGCATACGACAGTCTGAACACAGGAGGCTCAATCAG -1073

-1072 AACAGCTACTGAAGGCACATAAGACCCTGCTTCACTGTGGTGGCAGGC -1022

-1021 TGGGAAGAGGGAAGGCACGGAGGCTGCTGGCCTTCGACAAGACTTGGA -971

-970 AAAAGCTTTCCTGATTGGAGCAGGGCTGGAGGAGGGGAAGGGTCCATAA -920

-929 AGAATTCATAGCTCGGGAGAAGAAGGTGTATGTCCCATCCAGGTCACCCC -879

-878 GAGGCTTAGAGCCAAGACCCCAGTCTAGTTCCAGTCACAAACCTGACAC -828

-827 CATCAACTGAGGTCTCGTGAACACGGTGGCTGAAAGCATTTCTGTGTTTCC -777

-776 TGAGTCTCATTTATCCTCAGATCAACATGGGGACAAAGGCTTGAGGGACA -726

-725 AAGGCTTGAGGGACAAAGGCTTGAGGGACAAGGGTCTGTCTTTTGTTCTT -875

-674 TAAACAGAAGAGGAACGATGTTAGCCAGCCTGAGAAGGTGAAGCTCTGC -624

-623 CTGCTTCCACATGCCCCTGAGGCTTCCCCAAGGAGTCAGCCTGCTGGGGT -573

-572 TAGGGGGTTGCCCCGTGGAATTCCCCCAAATCCTGCTTCAAAGAGCCTGC -522

-521 TCCCAGAGGGCAGGAGAGGAGCTGAGTCAAAGACACTAACAGGGGGTG -471

-470 GGAATGAGAGGATAGAACCTACAGTGTGGGGAGGGGCTCCAGGCTGCCC -420

-429 TCTGGTCAGGGAGAGGGGTATCTCAGAAGCCCEAAGGAAGAGTGGTCTTG -379

-378 GGCTTCAGGTCTTCCAGTCCTATACAAGGCTGACCACTCAGGGCCTTCAG -328

-327 ACCTAGGACTAGATGGCCCCTCCACCATGCGTACCCTCCTCCTTTCCCA -277

-276 CAGATTCTGCAGTTIGCAAAAACTCAACCACTTCCCTGAGTGCTGTGGTTT -226

-225 CCTGTGGGTCTGGGGTCCTGCCTGACTTGGCAAGTGGGGGACTGTGGGC -175

-174 AGgGCATAAAGGAGTGGGTAGTGTAAGCACTTCTTTGGGCTGCCCAACA -124

-123 CACACACACACACACACACACACACACACACACACACACCCTGAGTCAGC $\quad-73$

-72 GTAAGCCTGGAGGGAGGGGCGGGGACACTGATTCAGTTTTCGTGCCTCI -22

-21 TIAAAACCTCCGCAAATTCTGCTTCACCCAGAAGCTTCGGTCTCACCATG +21

+22 AACCCCTGGCA

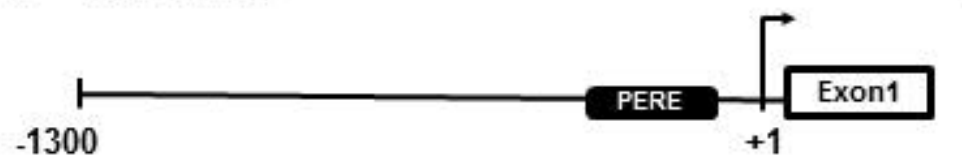

b

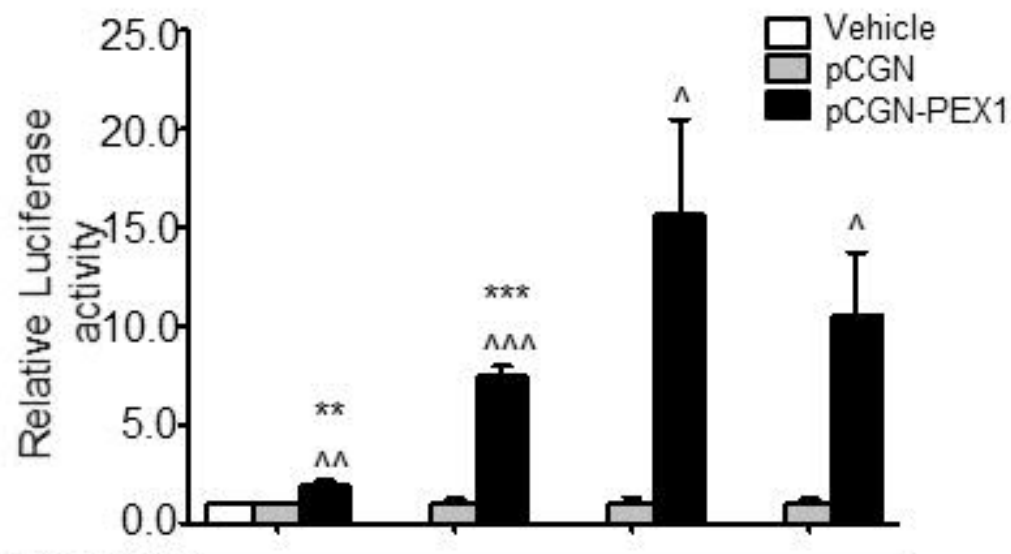

pGL-III-pMMP9 pCGN-PEX1 
Figure 8
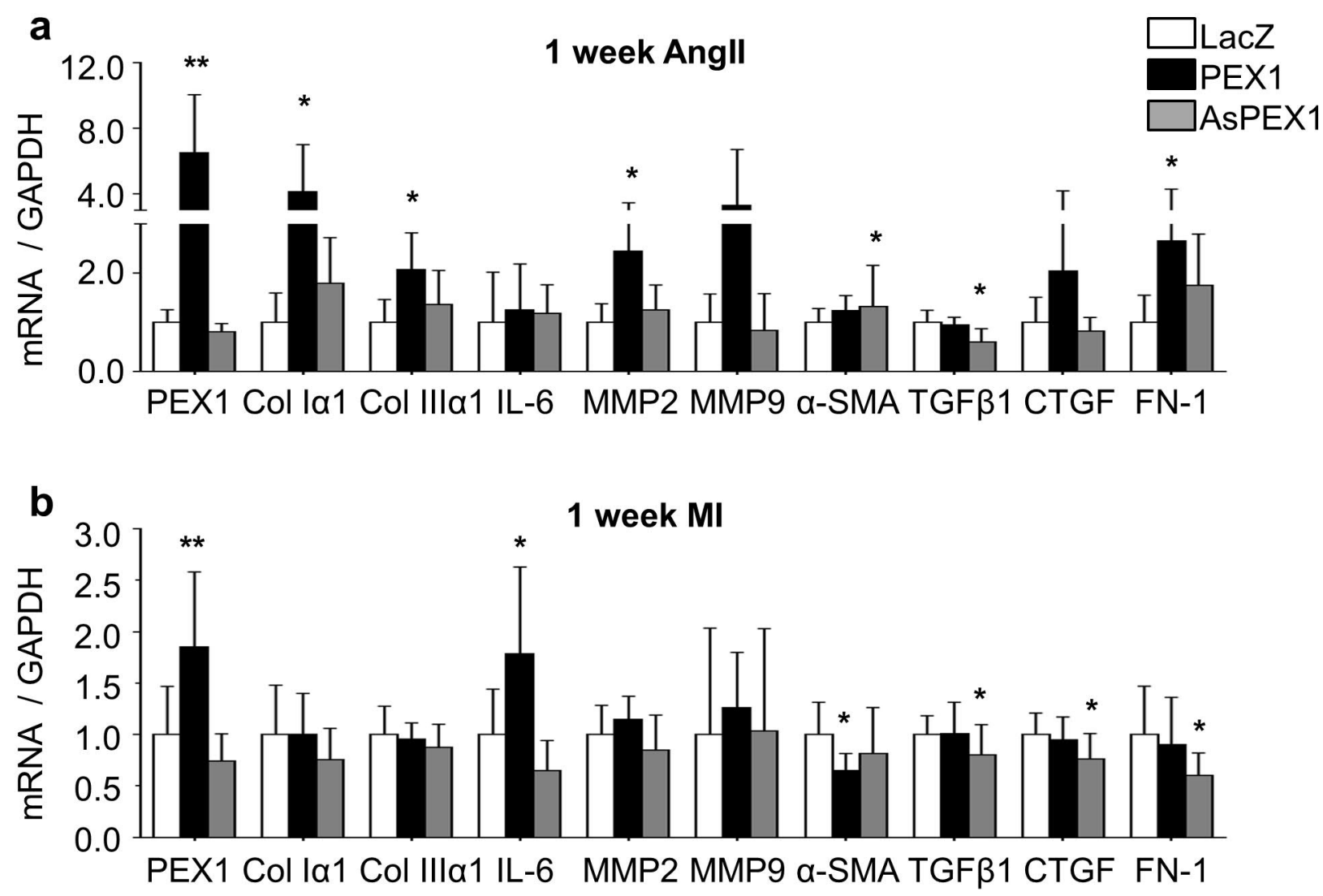
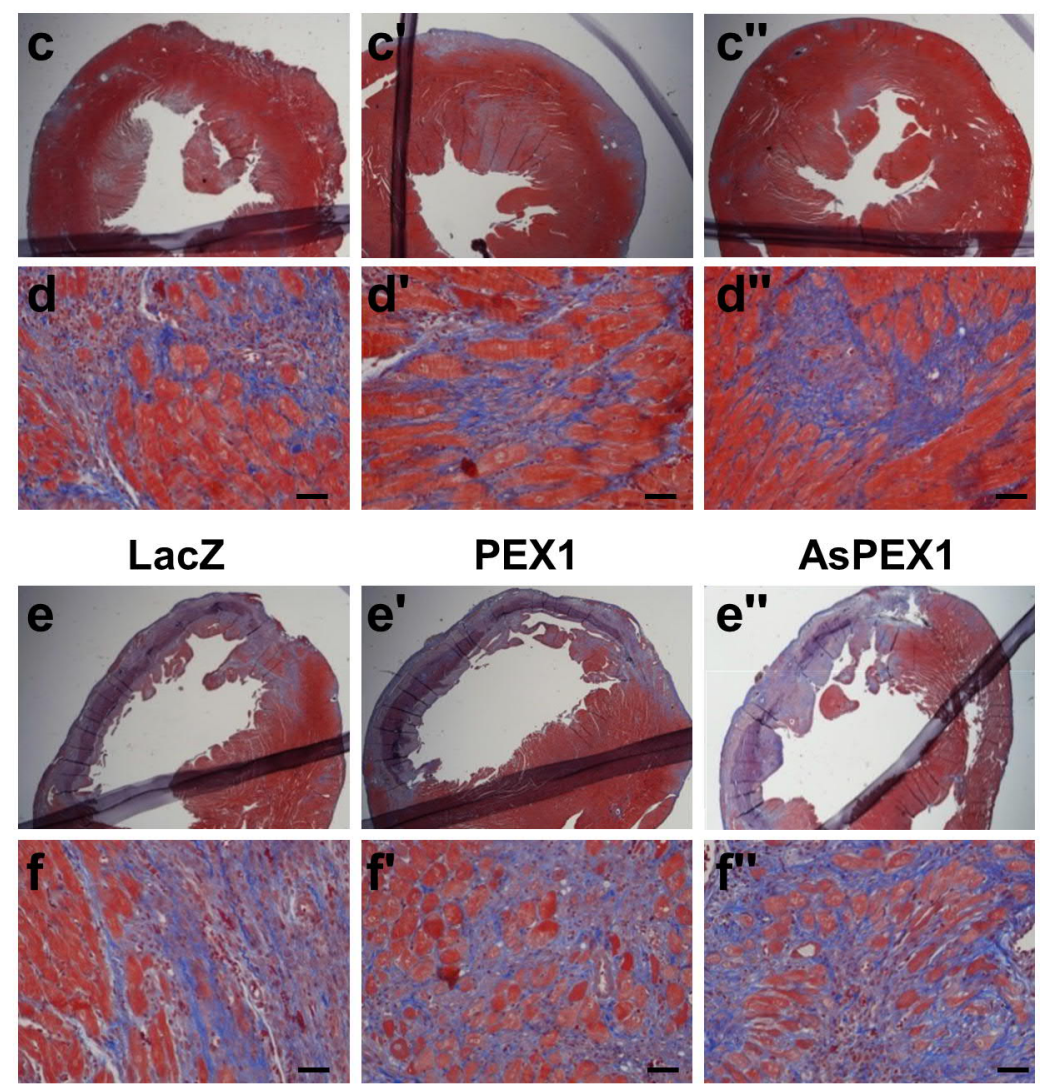

PEX1
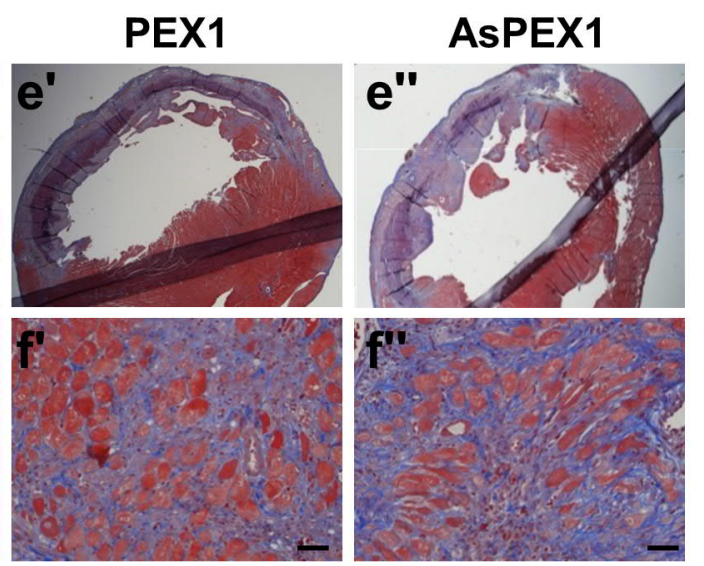
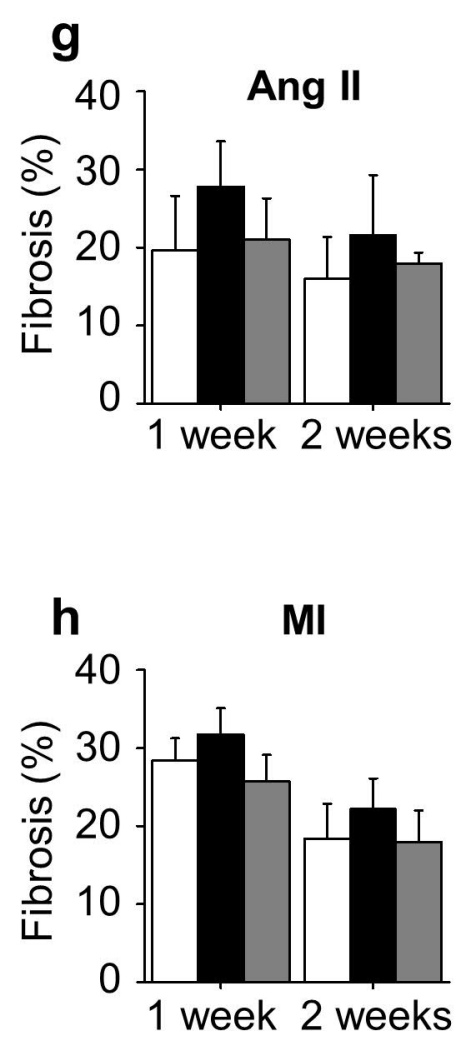
Figure 9

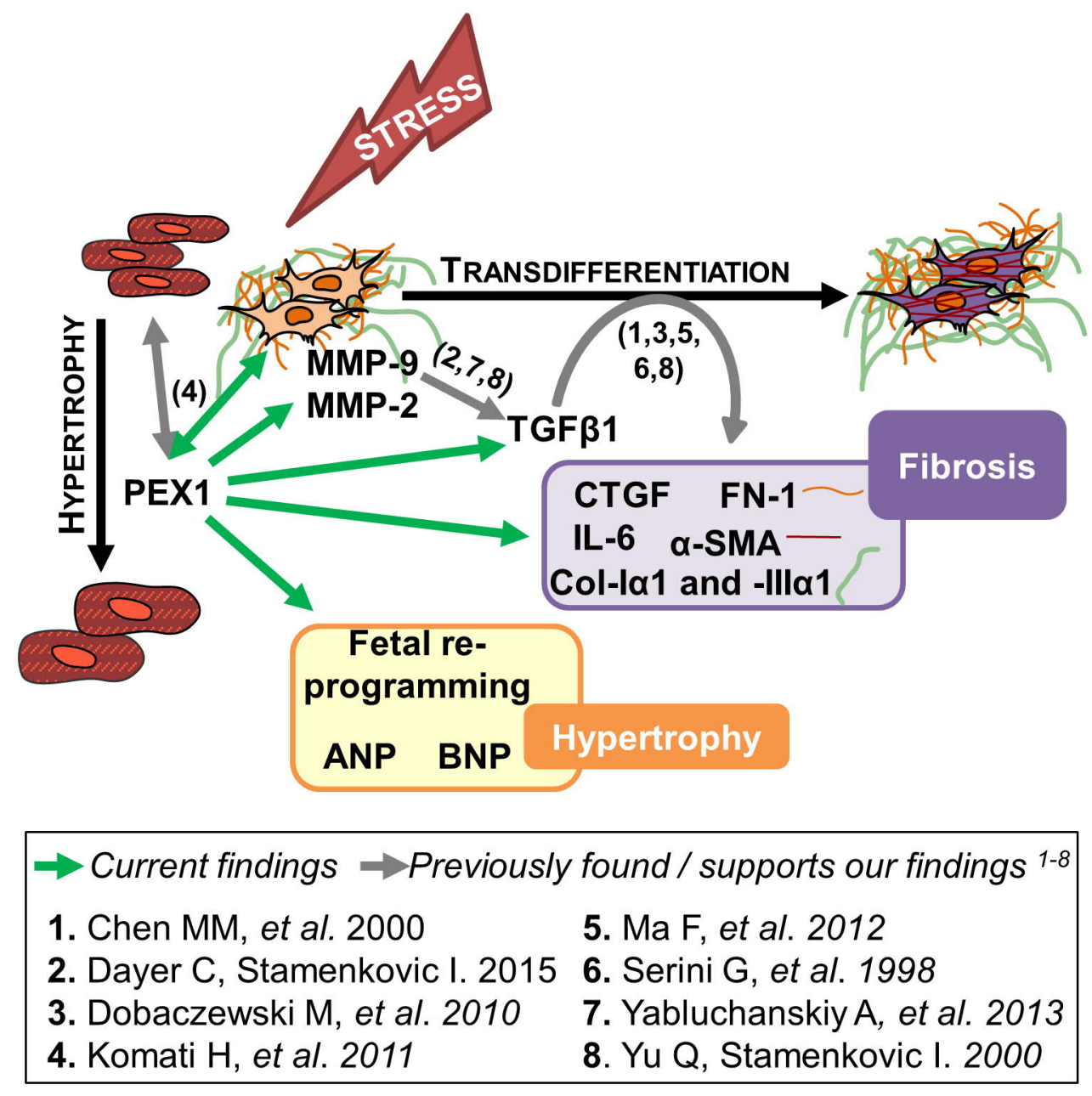




\title{
ELECTRONIC SUPPLEMENTARY MATERIALS
}

\section{Transcription factor PEX1 modulates extracellular matrix turnover through regulation of MMP-9 expression}

\author{
Alicia Jurado Acosta ${ }^{1}$, Jaana Rysä ${ }^{2}$, Zoltan Szabo ${ }^{1}$, Anne-Mari Moilanen ${ }^{3,4}$, Hiba Komati ${ }^{5}$, \\ Mona Nemer ${ }^{5}$, Heikki Ruskoaho ${ }^{1,6}$
}

1Department of Pharmacology and Toxicology, Research Unit of Biomedicine, Faculty of Medicine, University of Oulu, POB 5000, 90014 Oulu, Finland

${ }^{2}$ School of Pharmacy, Faculty of Health Sciences, University of Eastern Finland, POB 1627, 70211 Kuopio, Finland

${ }^{3}$ Cancer and Translational Medicine Research Unit, University of Oulu, POB 5000, 90014 Oulu, Finland

${ }^{4}$ Oulu University Hospital and Medical Research Center Oulu, POB 5000, 90014 Oulu, Finland

${ }^{5}$ Molecular Genetics and Cardiac Regeneration Laboratory, Department of Biochemistry, Microbiology and Immunology, University of Ottawa, 451 Smyth Road, Ottawa, Ontario, Canada K1H8M5

${ }^{6}$ Division of Pharmacology and Pharmacotherapy, Faculty of Pharmacy, University of Helsinki, POB 56, 00014 Helsinki, Finland

Corresponding author:

Heikki Ruskoaho, MD, PhD

heikki.ruskoaho@helsinki.fi 
Supplementary Table 1. Antibodies employed.

\begin{tabular}{lcccc}
\hline \multicolumn{1}{c}{ Antigen } & Product \# & Manufacturer & Detection & Application \\
\hline PEX1, Zfp260 & - & Debrus S, et al. MCB (2005) & Primary & IHC \& IHF \\
Prolyl-4-hydroxilase & MAB2073 & Millipore & Primary & IHF \\
Sarcomeric a-actinin & A7811 & Sigma-Aldrich & Primary & IHF \\
a-SMA & AB5694 & Abcam & Primary & IHF \\
WGA-FITC & W11261 & Thermofisher & Primary & IHC \\
Ki-67 & M7248 & Millipore & Primary & IHC \\
C-kit & sc-168 & Santa Cruz & Primary & IHC \\
vWF & AB7356 & Abcam & Primary & IHC \\
MMP-9 & AB76003 & Abcam & Primary & WB \\
Lamin B & SC-6216 & Santa Cruz & Primary & WB \\
GAPDH & MAB374 & Millipore & Primary & WB \\
AlexaFluor 568 anti-rabbit & A11036 & Invitrogen (Eugene, Oregon, US) & Secondary & IHF \\
Biotinylated anti-mouse IgG & BA-2001 & Vector Laboratories, Inc. (Burlingame, CA) & Secondary & IHF \\
Alexa Fluor 680 anti-mouse & A21058 & Invitrogen & Secondary & WB \\
Alexa Fluor 680 anti-rabbit & A21076 & Invitrogen & Secondary & WB \\
IrDye 700 anti-goat & $605-730-125$ & Rockland & Secondary & WB
\end{tabular}

PEX1 = phenylephrine-induced complex-1; $\alpha-S M A=$ alpha-smooth muscle actin; WGA-FITC $=$ wheat germ agglutinin/5'-fluorescein isothiocyanate; $v W F=$ von Willebreand factor; $M M P-9=$ metalloproteinase 9; GAPDH=glyceraldehyde 3-phosphate dehydrogenase; $\quad I H C=\quad$ Immunohistochemistry; $I H F=$ Immunohistofluorescence; $W B=$ Western blot 
Supplementary Table 2. Forward and reverse primer and fluorogenic probe sequences used for real time quantitative RT-PCR analysis.

\begin{tabular}{|c|c|c|}
\hline Gene & Primer & Fluorogenic probe \\
\hline ANP & $\begin{array}{l}\text { (F) GAAAAGCAAACTGAGGGCTCTG } \\
\text { (R) CCTACCCCCGAAGCAGCT }\end{array}$ & TCGCTGGCCCTCGGAGCCT \\
\hline a-SMA & $\begin{array}{l}\text { (F) TCCTGACCCTGAAGTATCCGATA } \\
\text { (R) GGTGCCAGATCTTTTCCATGTC }\end{array}$ & AACACGGCATCATCACCAACTGGGA \\
\hline$\alpha-\mathrm{MHC}$ & $\begin{array}{l}\text { (F) GCAGAAAATGCACGATGAGGA } \\
\text { (R) CATTCATATTTATTGTGGGATAGCAAC }\end{array}$ & TAACCTGTCCAGCAGAAAGAGCCTCGC \\
\hline BNP & $\begin{array}{l}\text { (F) TGGGCAGAAGATAGACCGGA } \\
\text { (R) ACAACCTCAGCCCGTCACAG }\end{array}$ & CGGCGCAGTCAGTCGCTTGG \\
\hline$\beta-\mathrm{MHC}$ & $\begin{array}{l}\text { (F) GCTACCCAACCCTAAGGATGC } \\
\text { (R) TCTGCCTAAGGTGCTGTTTCAA }\end{array}$ & TGTGAAGCCCTGAGACCTGGAGCC \\
\hline Acta1 & $\begin{array}{l}\text { (F) TCCTCCGCCGTTGGCT } \\
\text { (R) AATCTATGTACACGTCAAAAACAGGC }\end{array}$ & CATCGCCGCCACTGCAGCC \\
\hline Actc1 & $\begin{array}{l}\text { (F) GGGCCCTCCATTGTCCA } \\
\text { (R) GCACAATACTGTCGTCCTGAGTG }\end{array}$ & CGCAAGTGCTTCTGAGGCGGCTAC \\
\hline Col la1 & (F) CCCCTTGGTCTTGGAGGAA & $\begin{array}{l}\text { CTTTGCTTCCCAGATGTCCTATGGCTAT } \\
\text { GATG }\end{array}$ \\
\hline & (R) GCACGGAAACTCCAGCTGAT & \\
\hline Col IIla1 & $\begin{array}{l}\text { (F) AGCTGGCCTTCCTCAGACTTC } \\
\text { (R) GCTGTTTTTTGCAGTGGTATGTAATG }\end{array}$ & TTCCAGCCGGGCCTCCCAG \\
\hline CTGF & $\begin{array}{l}\text { (F) CGCCAACCGCAAGATTG } \\
\text { (R) CACGGACCCACCGAAGAC }\end{array}$ & CACTGCCAAAGATGGTGCACCCTG \\
\hline FN-1 & $\begin{array}{l}\text { (F) GCGAGGCAGGATCAGCTG } \\
\text { (R) CCAATCTTGTAGGACTGACCCC }\end{array}$ & ACCATTGCAAATCGCTGCCATGAA \\
\hline GAPDH & $\begin{array}{l}\text { (F) GGTCATCATCTCCGCCCC } \\
\text { (R) TTCTCGTGGTTCACACCCATC }\end{array}$ & CCGCTGATGCCCCCATGTTTG \\
\hline
\end{tabular}



IL-6
(F) CAGAATTGCCATTGCACAACTCTTTTCTCA
TGCATCATCGCTGTTCATACAA
(R) ATATGTTCTCAGGGAGATCTTGGAA

MMP-2 (F) CATGAAGCCTTGTTTACCATGG

TGGCAATGCTGATGGACAGCCC

(R) TGGAAGCGGAACGGAAACT

MMP-9 (F) CCGCCAACTATGACCAGGATAA

TGTATGGCTTCTGTCCTACTCGAGCCGA

(R) AGTTGCCCCCAGTTACAGTGA

PEX1 (F) TGAAGAGAGACCCAGTGAGTC

AACTCCCGTCCCCATGACAACCATC

(R) TTCCCGGCGTTTCTCTGAT

TGF $\beta 1$ (F) CATCGACATGGAGCTGGTGA

ACGGAAGCGCATCGAAGCCATC

(R) TTGGACAGGATCTGGCCAC

$18 S$

(F) TGGTTGCAAAGCTGAAACTTAAAG

CCTGGTGGTGCCCTTCCGTCA

(R) AGTCAAATTAAGCCGCAGGC

$\overline{A N P}$ and $B N P=A$-type and B-type natriuretic peptide; $\alpha$-SMA= alpha-smooth muscle actin; $M H C=$ myosin heavy chain; Acta1= skeletal alpha-actin; Actc1= cardiac alpha-actin; Col= collagen; CTGF= connective tissue growth factor, FN-1= fibronectin-1, GAPDH=glyceraldehyde 3-phosphate dehydrogenase, IL-6= interleukin-6, $M M P=$ metalloproteinase; $P E X 1=$ phenylephrine-induced complex-1; TGF $\beta 1=$ transforming growth factor $\beta 1, F=$ forward; $R=$ reverse. 


\section{Supplementary Figure Legends}

Fig. S1 a-SMA co-localizes with PEX1 in LV. Localization of PEX1 protein expression in myofibroblasts was evaluated by immunofluorescent co-staining with anti- $\alpha$-SMA antibody (a myofibroblast marker). Representative images are shown from LV of PEX1 treated hearts at day 3 after injection. White arrow heads indicate co-localization of the specific cell marker and PEX1 in the merged images (bottom right). The scale bar is $50 \mu \mathrm{m}$

Fig. S2 PEX1 overexpression leads to reactivation of the fetal gene program in adult rat heart. The mRNA levels of $\operatorname{ANP}(\mathbf{a}), \operatorname{BNP}(\mathbf{b}), \alpha-M H C(\mathbf{c}), \beta-M H C(\mathbf{d})$, the ratio of $\alpha-$ to $\beta-M H C$ isoforms $(\mathbf{e})$, $\operatorname{Actc1}(\mathbf{f}), \operatorname{Acta1}(\mathbf{g})$, and the ratio of $\operatorname{Actc1}$ to $\operatorname{Acta1}(\mathbf{h})$ were quantified by RT-qPCR and normalized to GAPDH. The results are expressed as mean \pm SD ( $N=5$ to 10 animals/group). ${ }^{*} \mathrm{P}<0.05$, ** $\mathrm{P}<0.01,{ }^{* *} \mathrm{P}<0.005$ versus LacZ control (Student's t-test or Mann Whitney U-test)

Fig. S3 Immunohistochemical evaluation of PEX1 gain- and loss-of-function revealed no significant changes in apoptosis, proliferation, number of stem cells or cell size. A) Representative images from 2 weeks normal rat heart histological sections are shown. The rate of apoptosis was assessed by TUNEL, immunohistochemical staining against Ki-67 was performed to study cellular proliferation, c-kit was used to stain stem cells, and to stain the cell extracellular membrane WGA was used. b-e Quantifications of TUNEL positive cells (b), dividing cells, indicated by Ki-67 marker (c), c-kit positive stem cells (d), and cross sectional area of myocytes using WGA (e) were performed by computerized methods ( $\mathrm{N}=6$ to 9 animals/group, c-e $\mathrm{N}=2$ for LacZ 1 week group). The results are expressed as mean $\pm S D$ and compared to LacZ control group (Student's t-test) 
Figure S1.
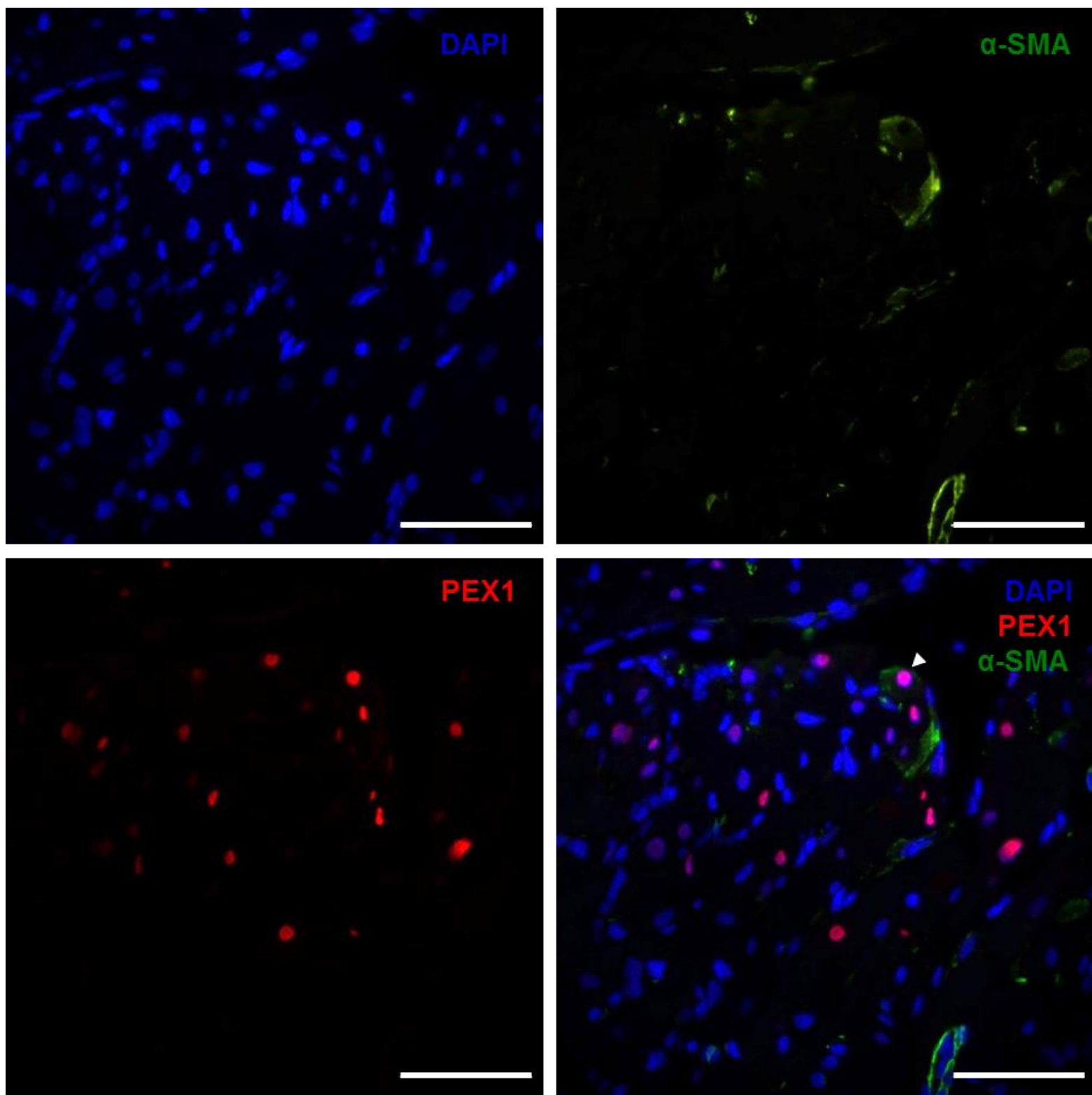
Figure S2.
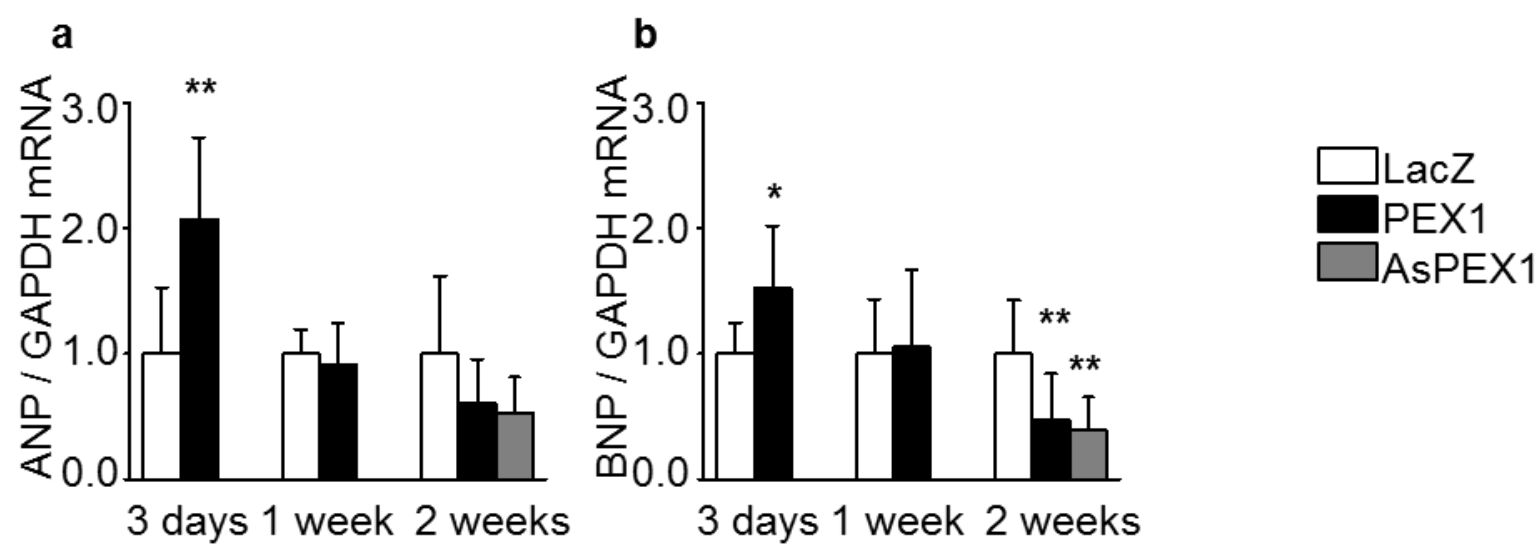

C
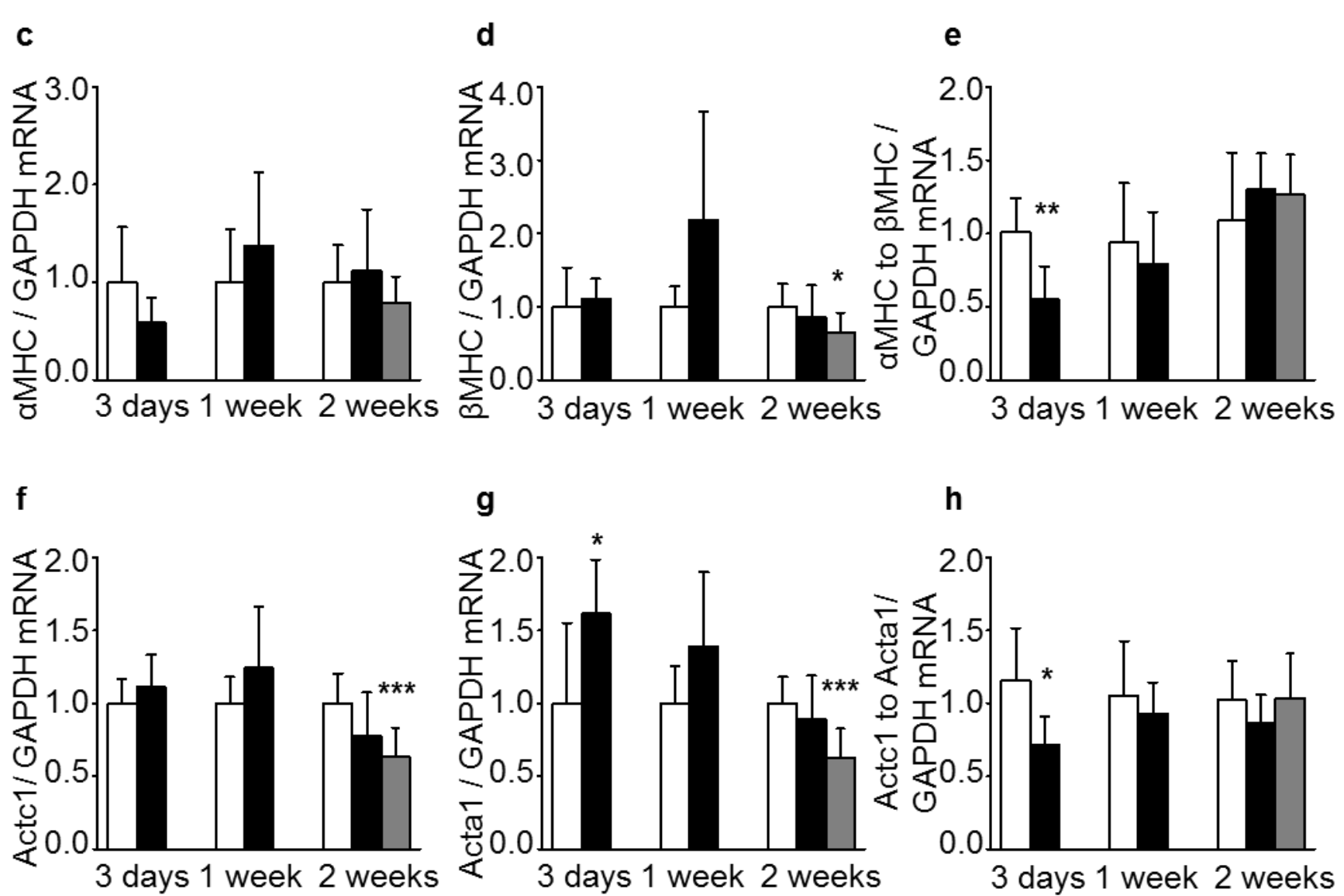

h
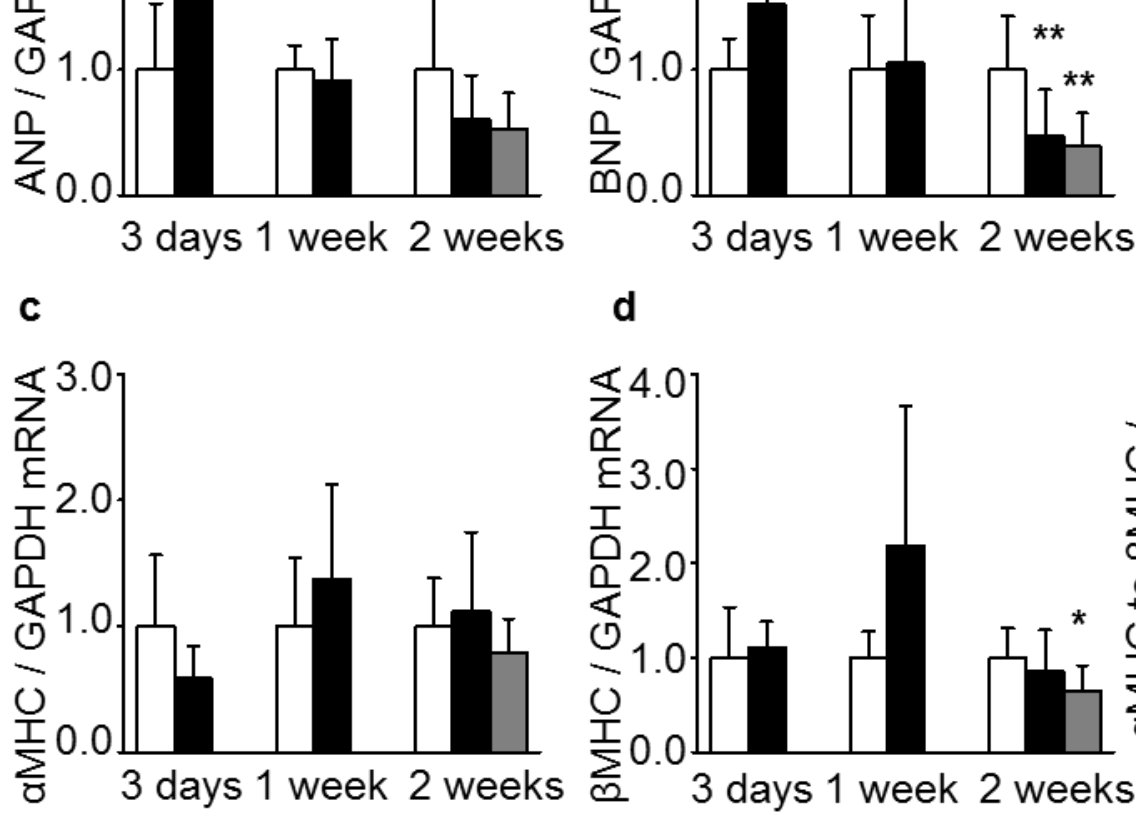

AsPEX1 
Figure S3.

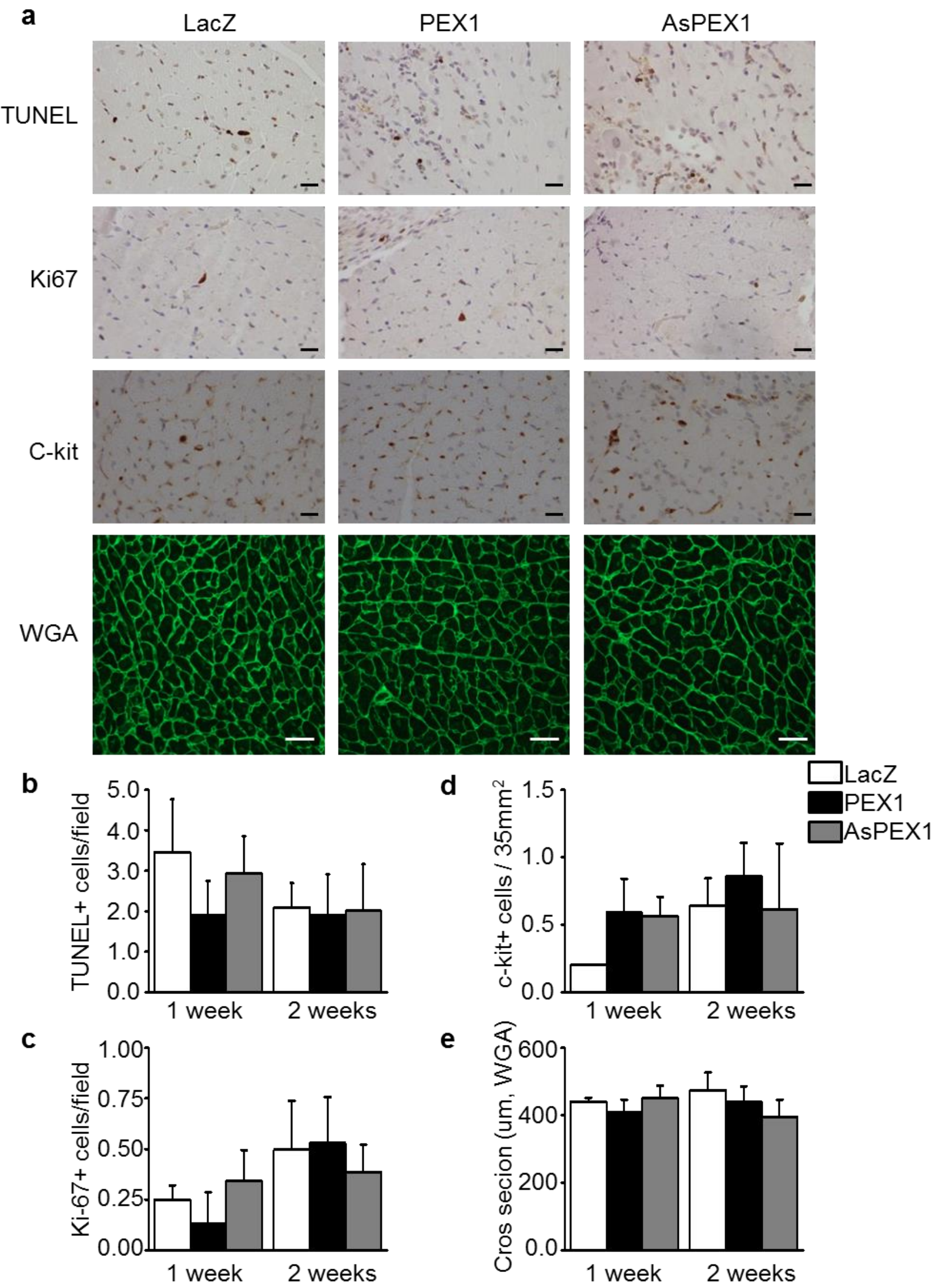

